In: Claus Ott/Hans-Bernd Schäfer (Hg.): Ökonomische Analyse des Unternehmensrechts. Beiträge zum

3. Travemünder Symposium zur ökonomischen Analyse des Rechts. Heidelberg: Physica, 1993,

S. $78-116$

\title{
Eine ökonomische Analyse der mitbestimmten Unternehmung
}

von Thomas Eger, Hans G. Nutzinger und Peter Weise

\section{A. Einleitung: Mitbestimmung als "Abschwächung" oder "Präzisierung" von Eigentumsrechten}

\section{Die theoretische Ausgangsfrage}

Es gibt verschiedene mögliche Zugänge zur Theorie der mitbestimmten Unternehmung. Zentral ist dabei die Frage, ob Mitbestimmung als Abschwächung von Eigentumsrechten (attenuation), als deren Umverteilung (redistribution) oder schließlich als Präzisierung dieser Rechte (specification) aufgefaßt wird. Je nachdem, für welche Betrachtungsweise man sich entscheidet, ergeben sich daraus unmittelbare und weitreichende Konsequenzen für die Art und Weise, wie die ökonomische Analyse der mitbestimmten Unternehmung durchgeführt wird.

Ein typisches Beispiel für den erstgenannten Ansatz im deutschsprachigen Raum ist das sog. "Kölner Gutachten" von Gerhard Prosi (1978). In diesem Gutachten, das im Auftrag des Bundesverbandes der Deutschen Arbeitgeberverbände für die vor dem Bundesverfassungsgericht anhängige Klage gegen das Mitbestimmungsgesetz 1976 erstellt wurde, vertrat der Verfasser im wesentlichen die Auffassung, durch die formal paritätische Besetzung des Aufsichtsrates (vgl. dazu unten Abschnitt II.) komme es zu einem "Durchschlag" dieser paritätischen Vertretung in diesem Kontrollgremium hin zu einer paritätischen Repräsentanz im Vorstand einer mitbestimmten Kapitalgesellschaft. Darüber hinaus führe die Verknüpfung von Unternehmensmitbestimmung nach dem Mitbestimmungsgesetz 1976 und von betrieblicher Interessenvertretung nach dem Betriebsverfassungsgesetz 1972 zu einer gefährlichen "Kumulation" dieser Mitbestimmungsmöglichkeiten, die zu Lasten unternehmerischer Entscheidungsfreiheit gehe. Schließlich identifiziert Prosi noch einen "Kaskadeneffekt", da Mitbestimmung in konzernverbundenen Gesellschaften sowohl im Aufsichtsrat der Obergesellschaft wie in den entsprechenden Kontrollorganen der Untergesellschaft stattfinde. Dadurch werde die Fähigkeit des Unternehmens, nach außen hin richtig auf die Signale des Marktes zu reagieren, entscheidend geschwächt, woraus sich sowohl betriebs- wie volkswirtschaftlich nachteilige Folgen ergäben.

Eine ähnliche, mehr in der Sprache der Eigentumsrechtstheorie formulierte Sichtweise vertritt auch S. Pejovich: Seiner Analyse zufolge werden durch Mitbestimmung 
Vertragsfreiheit und Recht auf Privateigentum, die beiden Grundvoraussetzungen einer freien Marktwirtschaft, eingeschränkt, und damit komme es zu einer Verschlechterung der Entscheidungen und zu einzel- wie auch gesamtwirtschaftlichen Effizienzverlusten. Im Anschluß an M. Jensen und W. Meckling sieht er den entscheidenden Beweis für die mutmaßlich negativen Folgen von Mitbestimmung - die er sowohl als Abschwächung wie auch als Umverteilung von Eigentumsrechten begreift - darin, daß diese partizipatorische Ausgestaltung der Eigentumsrechte, wäre sie wohlfahrtsverbessernd, sich ja von selbst durch freiwillige Vereinbarungen - ohne jeden gesetzlichen Druck - entwickelt hätte. Die schiere Tatsache, daß Mitbestimmungsregelungen qua Gesetz eingeführt wurden, dient den genannten Autoren schon als hinreichender Beleg dafür, daß diese rechtliche Normierung wohlfahrtsvermindernd sein $m u \beta$.

Gegen diese aprioristische Betrachtungsweise ist zumindest der folgende gravierende Einwand zu erheben: Das Argument einer naturwüchsigen Pareto-Verbesserung über wechselseitig vorteilhafte Verträge ist nur in einer Idealwelt ohne Informationsprobleme und Transaktionskosten zwingend, und in einer derartigen Welt würden sowohl der ideale Marktmechanismus durch "Abwanderung" als auch der ideale Mitbestimmungsmechanismus durch "Widerspruch" zu einem gleichermaßen effizienten Ergebnis führen. Das eigentlich zu behandelnde Problem, wie Eigentumsrechte aller im Unternehmen beteiligter Parteien zu definieren, zu spezifizieren und durchzusetzen sind, käme so gar nicht erst in den Blick (vgl. Eger, T./Weise, P.: Grundlagen; dieselben: Participation). Durch die Einführung der Mitbestimmung werden dann klar spezifizierte Rechte ohne Effizienzverlust umverteilt.

Geht man von einer derartigen idealisierten Marktvorstellung als Referenzmodell aus und vergleicht diese mit einer realen, Transaktionskosten verursachenden Mitbestimmung, so kann das Ergebnis jeglicher Form von Mitbestimmung nur eine Wohlstandsverschlechterung sein. Sie läuft in dieser Perspektive auf nichts anderes als auf die Einführung einer "künstlichen Beschränkung" - in der Sprache der Eigentumsrechtstheoretiker einer Abschwächung (attenuation) - unternehmerischer Entscheidungsfreiheit hinaus; und spätestens seit Paul A. Samuelsons Foundations of Economic Analysis (1947) wissen wir, daß sich nach dem LeChatelier-Prinzip die Anpassungsfähigkeit der Unternehmung an geänderte äußere Bedingungen um so mehr verringert, je größer die Zahl der Beschränkungen ist. Die von Prosi und anderen prognostizierten negativen Auswirkungen des Mitbestimmungsgesetzes sind im Grunde nichts anderes als eine (implizite) Anwendung dieses Prinzips. ${ }^{2}$

Geht man demgegenüber von der realen, unvollkommenen Welt mit Informations-

Das Bundesverfassungsgericht hat sich denn auch nicht von diesem Argumentationsmuster überzeugen lassen und sah sich angesichts widerstreitender Gutachten (zugunsten des Mitbestimmungsgesetzes hatte E. Kappler begutachtet) nicht in der Lage, eine "gesicherte Gesamtprognose" über die Auswirkungen dieses Gesetzes zu geben. Daher folgerte das Gericht, den Gesetzgeber treffe ein Korrekturgebot, wenn er sich mit seinen (günstigen) Prognosen geirrt haben sollte. "Damit wurde in augenfälliger Weise eine Verbindung zwischen der Verfassungsinterpretation einerseits, theoretischer und empirischer volkswirtschaftlicher Forschung andererseits, durch das Verfassungsgericht selbst hergestellt". (Backhaus, J., S. 111). 
problemen und positiven Transaktionskosten aus und vergleicht man Unternehmensverfassungen mit und ohne Mitbestimmung, so besteht die Alternative zu einer Unternehmensverfassung mit gesetzlich normierter Mitbestimmung nicht etwa in der unbeschränkten Handlungsfähigkeit des Unternehmens, sondern in anderen Formen, in denen sich dann Arbeitnehmerinteressen außermarktmäßig geltend machen können, sei es in Form häufiger Arbeitskonflikte, sei es durch rigide Kollektivvertragssysteme oder auch durch individualisierte "Protestaktionen", wie etwa Absentismus, Fluktuation, Leistungszurückhaltung bis hin zur Sabotage (vgl. etwa McCain, R.A.: Codetermination, $102 \mathrm{f}$.). Bei einer theoretischen wie auch empirischen Beurteilung der Konsequenzen gesetzlich verfaßter Unternehmensmitbestimmung ist also nicht eine Idealwelt ohne Beschränkungen, sondern eine ebenfalls unvollkommene Welt mit anderen Arten von Beschränkungen als Vergleichssituation in Rechnung zu stellen. Die Mitbestimmung ist dann daraufhin zu überprüfen, in welchem Maße sie zu einer Abschwächung, Umverteilung oder Präzisierung von Eigentumsrechten führt.

Es ist unmittelbar einsichtig, daß Mitbestimmung, soweit sie tatsächlich eine erhöhte Einflußnahme der Arbeitnehmer auf unternehmensbezogene und betriebliche Entscheidungen impliziert, in der Tat auch auf eine Umverteilung von Eigentumsrechten im weitesten Sinne hinausläuft. Aber diese Umverteilung ist nicht notwendig ein Nullsummenspiel, in dem der Kapitaleigner genau das verliert, was die Beschäftigten hinzugewinnen. ${ }^{3}$

Wir wollen im folgenden anhand einer Darstellung der rechtlichen Grundlagen und der theoretischen Überlegungen auf dem Hintergrund bisheriger empirischer Erfahrungen der Frage nachgehen, inwieweit Mitbestimmung eine möglicherweise wohlfahrtsverbessernde Präzisierung von Eigentumsrechten darstellen kann, die gerade aufgrund der Unbestimmtheit des Arbeitsvertrages hinsichtlich der konkret zu erbringenden Leistungen und der dabei implizierten Weisungsrechte der Unternehmensleitung zunächst einmal notwendig unspezifiziert geblieben sind. Wenn man, wie dies die in Abschnitt B dargestellten Unternehmenstheorien im Prinzip bei aller Verschiedenheit tun, die Unternehmung als eine Institution begreift, in der die Vorteile der Kooperation auch in solchen Situationen sichergestellt werden sollen, "in denen das Preissystem versagt" (Arrow, K.J., S. 33), dann muß man Mitbestimmung als ein mögliches Korrektiv für ein derartiges "Marktversagen" untersuchen. Mitbestimmung erscheint damit als eine der gesetzlichen Grundlagen dieser Kooperation im Unternehmen; ihre Beurteilung erfordert daher sowohl eine Klarstellung der oftmals vernachlässigten rechtlichen Normierungen als auch und vor allem eine empirische - und nicht nur apriorisch theoretische - Überprüfung ihrer möglichen ökonomischen Konsequenzen. Der vorliegende Beitrag versucht, dazu einige Vorüberlegungen in theoretischer und empirischer Hinsicht zu leisten. 


\section{Die wichtigsten mitbestimmungsrechtlichen Regelungen in Deutschland}

Da vor allem in der angelsächsischen Literatur zum Thema Mitbestimmung sehr stark auf die vermuteten Wirkungen einer Arbeitnehmerbeteiligung an den Entscheidungen des Unternehmens (insbesondere im Hinblick auf Investitionen, Einstellungen und Entlassungen) und die daraus möglicherweise resultierenden Konsequenzen für die Produktivität, die Kapitalrentabilität, die Höhe von Löhnen und Gewinnen in den mitbestimmten Unternehmen abgehoben wird, konzentriert sich der folgende Überblick über die wichtigsten rechtlichen Regelungen auf die Unternehmensebene und demzufolge vor allem auf das Mitbestimmungsgesetz von $1976 .{ }^{4}$ Vor allem in der US-amerikanischen Literatur wird Mitbestimmung häufig als "participation in management" (McCain, R.A.: Codetermination, S. 105) - also als Teilhabe an der Unternehmensführung - verstanden. Dieser Auffassung liegt implizit das US-amerikanische Konzept einer Gliederung der Unternehmensspitze in einen engeren "geschäftsführenden" und in einen erweiterten Vorstand (board of directors) zugrunde, wobei letzterer im Falle grundlegender Entscheidungen zusammentritt und die erforderlichen Beschlüsse faßt.

Dieses Modell ist aber nur äußerst bedingt vergleichbar mit der Zweiteilung in einen Vorstand und einen Aufsichtsrat nach dem deutschen Aktiengesetz: Nach diesem Modell obliegt die Geschäftsführung einzig und allein dem Vorstand, der nach $\S 76$ AktG die Gesellschaft leitet, ihre Geschäfte führt und sie gerichtlich und außergerichtlich vertritt; seine Vertretungsmacht kann nicht beschränkt werden ( $\$ 82 \mathrm{AktG}$ ). Demgegenüber ist der Aufsichtsrat für die Bestellung der Vorstandsmitglieder und ebenso für deren Abberufung aus wichtigem Grund ( $\S 84 \mathrm{AktG})$ zuständig. $\S 31$ MitbestG schreibt für die dem Mitbestimmungsgesetz von 1976 unterliegenden Unternehmen in der Rechtsform einer $\mathrm{AG}$, einer $\mathrm{KGaA}$, einer $\mathrm{GmbH}$, einer Bergrechtlichen Gewerkschaft oder einer Erwerbs- und Wirtschaftsgenossenschaft mit in der Regel mehr als 2.000 beschäftigten Arbeitnehmern ( $\$ 1$ MitbestG) generell die Bildung eines solchen Aufsichtsrates vor.

Dieser Aufsichtsrat ist jedoch als Kontroll-, nicht als Entscheidungsorgan konzipiert: Nach § 111(1) AktG hat er die Aufgabe, die Geschäftsführung zu überwachen, und damit ihm dies möglich ist, werden dem Aufsichtsrat als Kollektivorgan weitgehende Informationsrechte zugestanden ( $\S 111(2) \mathrm{AktG})$. Darüber hinaus haben auch die einzelnen Aufsichtsratsmitglieder nach $\S 90$ AktG umfangreiche Auskunftsrechte, die ein einzelnes Mitglied sogar gegen den Vorstand durchsetzen kann, wenn es von einem Gewerkschaften nach dem Montan-Mitbestimmungsgesetz 1951 gehen wir angesichts der zahlenmäßig geringen und weiter abnehmenden Bedeutung dieses Bereichs (Stahlerzeugung und Kohlebergbau mit derzeit noch knapp 300.000 Beschäftigten) nicht weiter ein. Ebenso wird die in Kapitalgesellschaften zwischen 500 und 2.000 Beschäftigten bestehende Drittelbeteiligung von Arbeitnehmer- und Gewerkschaftsvertretern nach dem Betriebsverfassungsgesetz1952 hier nicht weiter verfolgt. 
zweiten Aufsichtsratsmitglied unterstützt wird ( $\$ 90(3)$ AktG). Andererseits bestimmt das Aktiengesetz aber auch unmißverständlich, daß dem Aufsichtsrat Maßnahmen der Geschäftsführung nicht zustehen (§ 111(4) AktG).

Abgesehen von den weitgehenden Informationsrechten, die dem Aufsichtsrat als Kollektivorgan, aber auch einzelnen Mitgliedern zustehen, damit die gesetzlich vorgesehene Kontrollaufgabe effektiv wahrgenommen werden kann, gibt es aber nur sehr eingeschränkte Entscheidungsrechte, die sich im wesentlichen auf die bereits erwähnte Bestellung und Abberufung der Vorstandsmitglieder beziehen. Nicht vergleichbar mit dem amerikanischen erweiterten Vorstandsmodell ist dagegen die Möglichkeit, daß wichtige Geschäfte nach $\S 111(4)$ von der Zustimmung des Aufsichtsrats abhängig gemacht werden können, sei es durch Satzung des Unternehmens, sei es durch die Geschäftsordnung des Aufsichtsrats oder auch durch einen auf den Einzelfall bezogenen Aufsichtsratsbeschluß. Hier kommt dem Aufsichtsrat, anders als dem erweiterten board of directors, kein Entscheidungs-, sondern allenfalls ein Vetorecht zu. Aber selbst dieses Vetorecht eines (mitbestimmten) Aufsichtsrates ist nicht unbeschränkt: "Auch diese mögliche Ausübung von Arbeitnehmerinteressen stößt jedoch an Grenzen. Wenn der Aufsichtsrat die Zustimmung zu einem Geschäft des Vorstands verweigert, kann dieser die Hauptversammlung anrufen, die dann mit Dreiviertelmehrheit die Zustimmung erteilen kann ( $§ 111$ Abs. 4 Sätze 3-5 AktG). Durch dieses Letztentscheidungsrecht kann die Aufsichtsratsmitbestimmung unterlaufen werden" (Nagel, B.: Unternehmensmitbestimmung, S. 53 f.).

Wie sieht nun die zahlenmäßige Beteiligung der Arbeitnehmer im Aufsichtsrat bzw. im Vorstand einer (mitbestimmungspflichtigen) Kapitalgesellschaft aus? Wie bereits oben angedeutet, gilt das Mitbestimmungsgesetz von 1976 für alle Kapitalgesellschaften typischerweise in der Rechtsform der AG oder der GmbH - mit mehr als 2.000 Beschäftigten, ausgenommen die wenigen verbliebenen Unternehmungen des Montanbereichs, in denen das Montan-Mitbestimmungsgesetz von 1951 weiterhin gilt, und unter Ausschluß der Tendenzunternehmen und Einrichtungen der Religionsgemeinschaften. ${ }^{5}$ Insgesamt werden derzeit (1990) 544 Kapitalgesellschaften erfaßt, davon 321 AGs und 202 GmbHs. Diese repräsentieren nach allen ökonomischen Kriterien wie Beschäftigung, Umsatz, Wertschöpfung und Unternehmenskapital - einen erheblichen Anteil an der bundesdeutschen Wirtschaft; die Schwerpunkte liegen in den Bereichen Metall, Handel-Banken-Versicherungen, Chemie-Papier-Keramik und Öffentliche Dienste, Transport und Verkehr. ${ }^{6}$

Formal ähneln die Regelungen des Mitbestimmungsgesetzes 1976 weitgehend den entsprechenden Vorschriften des Montanmitbestimmungsgesetzes von 1951, aber im Betriebsverfassungsgesetz 1972 eingeschränkt, für Einrichtungen der Religionsgemeinschaften sogar ganz ausgeschlossen ( $\$ 118(2)$ BetrVG). ÖTV (546). Vgl. Informationsdienst des Instituts der deutschen Wirtschaft, S. 2. 
Vergleich dazu sind in allen entscheidenden Fragen die Rechte der Arbeitnehmerseite und der Gewerkschaften eingeschränkt. Der wesentliche Unterschied ist dabei in der Zusammensetzung des zentralen Kontrollorgans der mitbestimmten Kapitalgesellschaft, also des Aufsichtsrates, zu sehen; seine Größe hängt von der Beschäftigtenzahl ab. ${ }^{7}$ Das Mitbestimmungsgesetz von 1976 hält, insoweit dem alten Montan-Mitbestimmungsgesetz folgend, zumindest formal am Prinzip der Parität fest; faktisch ergibt sich jedoch ein Übergewicht der Anteilseignerseite. Dies läßt sich leicht am Beispiel der kleinsten Größenklasse - zwischen 2.000 und 10.000 Beschäftigte - veranschaulichen: Im Regelfall (vgl. Fußnote 7 oben) hat der Aufsichtsrat dann zwölf Mitglieder, von denen insgesamt sechs Mitglieder die Arbeitnehmerseite repräsentieren. Zwei Arbeitnehmervertreter werden von den zuständigen Gewerkschaften nominiert, und vier Mitglieder vertreten die vom Gesetz vorgesehenen Gruppen von Beschäftigten, nämlich Arbeiter, Angestellte und leitende Angestellte. Da jede Gruppe Anspruch auf mindestens einen Sitz im Aufsichtsrat hat, begünstigt der so gewährte "Minderheitenschutz" natürlich in der Praxis die Repräsentanten der leitenden Angestellten, die auf jeden Fall mindestens einen Vertreter in den Aufsichtsrat entsenden können. Diese Sondervertretung der leitenden Angestellten und die damit zusammenhängende Aufteilung der Belegschaft in drei statt zwei Gruppen macht einen wichtigen Unterschied zum Betriebsverfassungsgesetz aus, das seinerseits gerade die leitenden Angestellten von der aktiven und passiven Wahl zu den Vertretungsorganen der Arbeitgeberseite (Betriebsrat usw.) ausschließt (§ 5(3) BetrVG 1972).

Bekanntermaßen war die Sondervertretung der leitenden Angestellten in den ursprünglichen Gesetzentwürfen nicht vorgesehen, sondern das Ergebnis eines langen koalitionspolitischen Ringens zwischen FDP und SPD in der damaligen Bundesregierung (vgl. Nagel, B.: Unternehmensmitbestimmung, S. 93-97). Die Einführung eines dritten Faktors "Disposition" durch den liberalen Koalitionspartner, der - bei formaler Wahrung der Parität - dann aber der Arbeitnehmerseite zugerechnet wurde, bedeutet - auch nach den empirischen Erfahrungen (vgl. Nagel, B.: Unternehmensmitbestimmung; Nagel, B.: Paritätische Mitbestimmung; Bamberg U. et al.) - zweifellos eine indirekte Stärkung der Kapitaleignerseite, da die Vertreter der leitenden Angestellten, obwohl der Arbeitnehmerseite voll zugerechnet, sich zumindest teilweise auch als Vertreter der Unternehmensleitung verstehen. Schon aus diesem Grunde kann selbst im Kontrollgremium Aufsichtsrat keine vollständige Gleichberechtigung von "Arbeit und Kapital" erwartet werden.

Diese Aufspaltung der Belegschaft in drei verschiedene Arbeitnehmergruppen führt nun zu einem äußerst komplizierten Wahlverfahren (\$§ 8-24 MitbestG), das aus der Sicht der Gewerkschaften entsolidarisierend wirkt und daher eine weitere Schwächung der Arbeitnehmerseite bedeutet. Die Vorschriften des Gesetzes sehen für Arbeiter und

Nach $\S 7$ MitbestG setzt sich der Aufsichtsrat eines mitbestimmten Unternehmens mit in der Regel unter 10.000 Arbeitnehmern aus je sechs Aufsichtsratsmitgliedern der Anteilseigner und der Arbeitnehmer zusammen; die Zahlen erhöhen sich auf jeweils acht bei 10.000 bis 20.000 Beschäftigten und auf jeweils zehn bei mehr als 20.000 Arbeitnehmern. Durch Satzung des Unternehmens ist eine Erhöhung der Zahl der Aufsichtsratsmitglieder von sechs auf acht oder zehn bzw. von acht auf zehn zulässig. 
Angestellte und in bestimmten Fällen sogar für die leitenden Angestellten getrennte Wahlverfahren vor. Überdies ist in allen Unternehmen mit mehr als 8.000 Beschäftigten ein indirektes Wahlverfahren mit Wahlmännern vorgesehen ( $\$ 9$ MitbestG). ${ }^{8}$

Eine weitere Abschwächung erfährt die Position der Arbeitnehmerseite bei der Wahl des Aufsichtsratsvorsitzenden, der anders als in $\S 9$ MontanmitbestG kein weiteres, "neutrales" Mitglied mehr ist: Erhält bei der Wahl zum Aufsichtsratsvorsitzenden kein Kandidat eine Zweidrittelmehrheit, so wird der Aufsichtsratsvorsitzende mit der Mehrheit der Stimmen der Anteilseignervertreter gewählt. ${ }^{9}$ Dementsprechend ist in aller Regel der stellvertretende Aufsichtsratsvorsitzende ein Vertreter der Arbeitnehmerseite, wobei etwa $80 \%$ dieser Stellvertreter Mitglieder einer DGB-Gewerkschaft sind. Der Aufsichtsratsvorsitzende - nicht jedoch sein Stellvertreter - hat nach dem Gesetz bei Patt-Situationen ein Doppelstimmrecht, so daß selbst bei einer gemeinsamen einheitlichen Stimmabgabe aller Arbeitnehmervertreter (einschließlich des Repräsentanten der leitenden Angestellten) im Konfliktfall die Anteilseignerseite eine Mehrheit hat.

Diese zwar formal paritätische, aber materiell doch schwächere Beteiligung der Arbeitnehmerseite schlägt auch bei der Bestellung des Vorstandes durch; dies gilt sowohl für die gesetzlichen Regelungen als auch für die praktischen Erfahrungen nach nunmehr fünfzehn Jahren Geltung dieses Gesetzes. Zwar sieht § 31(2) MitbestG eine Zweidrittelmehrheit für die Bestellung der Vorstandsmitglieder vor; kommt diese jedoch nicht zustande, so genügt eine einfache Mehrheit, gegebenenfalls mit dem Doppelstimmrecht des (im Regelfall der Anteilseignerseite zuzurechnenden) Aufsichtsratsvorsitzenden.

Diese Stimmrechtsregelung hat nun unmittelbare Konsequenzen für die Bestellung des für Personalwesen zuständigen Vorstandsmitglieds, des Arbeitsdirektors: Während dieser nach $\S 13$ MontanmitbestG nicht gegen die Stimmen der Mehrheit der Arbeitnehmervertreter im Aufsichtsrat gewählt werden kann (und damit auch regelmäßig der Arbeitnehmerseite zuzurechnen ist), findet sich eine entsprechende Schutzvorschrift für die Bestellung des Arbeitsdirektors nicht in $\S 33$ MitbestG. Dies hat im Regelfalle - vor allem außerhalb des öffentlichen Sektors - dazu geführt, daß Kandidaten der Anteilseignerseite für diese Vorstandsposition gewählt wurden, im Konfliktfalle sogar mit dem Doppelstimmrecht des Aufsichtsratsvorsitzenden (vgl. Bamberg U. et al., S. 191-216). Hierbei ist ein empirischer Befund bemerkenswert: Eine formal gleichlautende Vorschrift in dem ebenfalls für den Montanbereich geltenden Mitbestimmungsergänzungsgesetz von 1956 hat in den betroffenen Konzernen nichts daran geändert, Abhaltung der Wahlen sehr viel Zeit in Anspruch nahm und auch mit erheblichen Rechtsstreitigkeiten zwischen Unternehmen und Gewerkschaften verbunden war. $\mathrm{Zu}$ den ersten Erfahrungen vgl. im einzelnen Nagel, B.: Unternehmensmitbestimmung, S. 89-107, sowie Bamberg, U. et al., S. 95-122. vorsitzenden zu 85 - $90 \%$ bereits im ersten Wahlgang besetzt wurde (vgl. Bamberg, U. et al., S. 111). 
daß der Arbeitnehmer- und Gewerkschaftsseite faktisch eine Art "Zugriffsrecht", wie in den montanmitbestimmten Unternehmen, zugestanden wurde. Die daran geknüpfte Erwartung des Bundesministers für Arbeit und Sozialordnung, die mit dem Mitbestimmungsergänzungsgesetz gemachten Erfahrungen berechtigten "zu der Annahme, daß im Interesse des Unternehmens und entsprechend dem Sinn und Zweck des Mitbestimmungsgesetzes Personen zu Arbeitsdirektoren bestellt werden, die in besonderem Maße das Vertrauen der Arbeitnehmerseite genießen" (S. 47), hat sich - außerhalb des Bereichs öffentlicher oder gemischtwirtschaftlicher Unternehmen - kaum bestätigt. Bamberg U. et al. (S. 209) bewerten die Erfahrungen mit den Arbeitsdirektoren nach dem Mitbestimmungsgesetz 1976 dahingehend, daß diese in der Regel eine "distanzierte Kooperation mit Arbeitnehmervertretern" pflegten (vgl. dazu unten Abschnitt D.).

Wie bereits eingangs betont, haben wir bewußt die Regelungen des Mitbestimmungsgesetzes von 1976 und verwandte Vorschriften in den Vordergrund dieses Überblicks über rechtliche Regelungen gestellt. Es soll aber schon an dieser Stelle darauf hingewiesen werden, daß die praktischen Wirkungen des Mitbestimmungsgesetzes in engem Zusammenhang mit der Funktion des Betriebsratsvorsitzenden und des Wirtschaftsausschusses zu sehen sind, also mit Personen bzw. Institutionen, deren Aufgaben durch das Betriebsverfassungsgesetz von 1972 festgelegt werden.

\section{B. Ökonomische Analyse der Koordination von Entscheidungen in der Unternehmung}

\section{Vier Grundtypen von Unternehmungstheorien}

Wir haben bei unserer Darstellung der rechtlichen Grundlagen von Unternehmensmitbestimmung den Begriff "Unternehmung" in einem allgemeinen Sinne verwendet, ohne ihn näher zu spezifizieren. Vom Standpunkt der ökonomischen Theorie können wir diese Organisationen zunächst einmal folgendermaßen charakterisieren: Unternehmungen sind Einheiten, bestehend aus einer Person oder mehreren Personen, in denen produziert wird, d. h. Güter in andere Güter umgewandelt werden. Die Frage ist nun aber: Welcher Koordinationsmechanismus koordiniert die Produktionstätigkeiten der Personen innerhalb der Unternehmung, und warum wird der Marktmechanismus bei den unternehmensinternen Aktivitäten durch einen anderen Mechanismus ersetzt? ${ }^{10}$ Auf diese Frage gibt es eine Vielfalt von Antworten, die sich indessen auf vier Grundtypen zurückführen lassen. ${ }^{11}$

11 Vgl. zum folgenden Weise, P. et al. Zum Vergleich von Markt und Institutionen allgemein s. Weise, P./Brandes, W., TaD. 
Die Team-Theorie von Alchian und Demsetz (1972): Diese Theorie begründet die Existenz der Unternehmung mit der These, daß mehrere Personen, die gemeinsam in einem Team produzieren, einen höheren Ertrag haben, als wenn diese Personen jede für sich allein produzieren. Wenn jede Person für sich allein etwas herstellt, kann man ihr dieses Produkt zurechnen, und sie kann es auf dem Markt verkaufen; wenn aber mehrere Personen in einem Team etwas arbeitsteilig herstellen, kann keiner einzelnen Person ein verkaufsfähiges Einzelprodukt zugerechnet werden. Wenn es aber schwierig oder unmöglich ist, den Effekt der Arbeit jeder einzelnen Person an dem Gesamteffekt des Teams festzustellen, hat jedes Teammitglied einen Anreiz, sich vor zu großer Anstrengung zu drücken - es bummelt und schont sich für den Feierabend -, in der Hoffnung, daß die anderen arbeiten. Denkt aber jeder so, entfallen die Vorteile der Teamproduktion; wir haben ein typisches Gefangenen-Dilemma-Spiel vor uns, mit den Alternativen "bummeln" und "hart arbeiten" und mit der kollektiv-rationalen Lösung der harten Arbeit für alle. Um sicherzustellen, daß auch wirklich alle arbeiten, muß es Überwacher und Aufsichtspersonen geben, die im wohlverstandenen Interesse der Arbeiter diese beaufsichtigen, ihnen die individuellen Leistungen zuzurechnen versuchen und sie daran hindern zu bummeln. Sind die Überwachungskosten geringer als der Vorteil der Teamproduktion gegenüber den Einzelproduktionen, ist es im Interesse der Arbeiter und der Aufsichtspersonen, sich freiwillig in einem Team zusammenzuschließen, da alle gewinnen können - die Existenz der Unternehmung ist erklärt.

Die Unternehmung ist also ein Mittel zur Organisation und Beaufsichtigung von Teamproduktion. Die Unternehmung ist ein Substitut für Wettbewerbsmärkte. Die disziplinierenden Effekte des Wettbewerbs werden in die Unternehmung übertragen und dort von den Aufsichtspersonen bewirkt. Aber wer überwacht die Überwacher? Diese haben zum einen einen Anreiz, gut zu überwachen, weil sie sich in Konkurrenz zu anderen Aufsichtspersonen befinden; sie haben zum anderen dann einen Anreiz, gut zu überwachen, wenn sie mit dem Überschuß des Ertrages aus der Teamproduktion nach Abzug der festgelegten Entlohnungen für die anderen Teammitglieder, sprich: die Arbeiter, entlohnt werden. Sie erhalten also die Gewinne oder Verluste - damit finden Unternehmer und Manager ihre Erklärung.

Der Beaufsichtiger, sprich: der Unternehmer, hat das alleinige Recht, Mitglieder zu heuern oder zu feuern; so kann er disziplinieren. Er ist auch der gemeinsame Vertragspartner für alle Teammitglieder; nur mit ihm werden Arbeitsverträge ausgehandelt, untereinander schließen die Teammitglieder keine Verträge ab. Diese Verträge werden von den Arbeitern freiwillig mit dem Unternehmer abgeschlossen, da sie ja alle nur gewinnen können. Die Beziehungen innerhalb der Unternehmung sind folglich rein vertraglicher Natur: Wenn der Arbeiter A etwas tun soll und dies auch tut, so hat er mit dem Beaufsichtiger in diesem Moment einen Vertrag abgeschlossen - zwar keinen expliziten, aber einen impliziten Vertrag -, hat aber keinem Befehl Folge geleistet. Der Unterschied zwischen Markt und Unternehmung ist demnach lediglich darin zu sehen, daß auf dem Markt jeweils zwei Personen einen Vertrag schließen, während in der Unternehmung eine zentrale Vertragspartei existiert, mit der alle Teammitglieder freiwillig explizite und implizite Verträge abschließen - die hierarchische Unternehmungsorganisation, wenn auch auf freiwilliger Basis, ist begründet. 
Die Transaktionskostentheorie von Coase (1937): Diese Theorie begründet die Existenz der Unternehmung mit der These, daß die Benutzung des Marktes Kosten verursacht und daß die Einrichtung einer Unternehmung diese Kosten vermeiden kann. Die Kosten, die mit dem Markt- und Preismechanismus verbunden sind, heißen Transaktionskosten, weil sie sich auf die Kosten einer Transaktion, d. h. die Durchführung einer Handlung, beziehen. Im einzelnen sind dies: Such- und Informationskosten, Verhandlungskosten und Kontrollkosten. Diese Kosten können gesenkt werden, wenn eine nicht-marktliche Organisationsform gebildet wird, in der einem Unternehmer die Macht übertragen wird, die Produktionshandlungen zu lenken. Die Arbeiter schließen mit dem Unternehmer einen Vertrag ab, in dem sie sich verpflichten, den Befehlen des Unternehmers Folge zu leisten, und in dem der Unternehmer sich verpflichtet, einen Lohn zu zahlen. Dieser Vertrag ist die Grundlage für die Befehlshoheit des Unternehmers; der Vertrag schränkt diese Befehlshoheit aber gleichzeitig auf bestimmte Tätigkeiten ein.

Das charakteristische Merkmal einer Unternehmung liegt für Coase darin, daß Überund Unterordnungsverhältnisse bestehen, daß die einzelnen Aktivitäten innerhalb der Unternehmung durch Befehl und Gehorsam koordiniert werden. Während bei marktlicher Koordination ein Produktionsfaktor auf Preisveränderungen reagiert und beispielsweise aus einem Wirtschaftsbereich $\mathrm{Y}$ in einen Wirtschaftsbereich $\mathrm{X}$ wandert, weil dort der erzielte Lohn höher ist, so ist dies in der Unternehmung anders: "Wenn ein Arbeiter von der Abteilung $\mathrm{Y}$ in die Abteilung $\mathrm{X}$ geht, so tut er das nicht wegen einer Veränderung der relativen Preise, sondern weil es ihm aufgetragen wurde."

Bis zu einer bestimmten Unternehmungsgröße ist es kostengünstiger, Transaktionen durch Befehl und Gehorsam innerhalb einer Unternehmung durchzuführen als über den Markt. Es werden also die Kosten der Nutzung des Markt- und Preismechanismus mit den Kosten der Koordination innerhalb einer Unternehmung verglichen. Eine optimale Unternehmungsgröße, d. h. auch ein optimales Verhältnis von Markt und Hierarchie, ist dann erreicht, wenn die Kosten der Organisation einer zusätzlichen Transaktion innerhalb der Unternehmung den Kosten einer zusätzlichen Markttransaktion entsprechen.

Die Unsicherheits-Theorie von Knight (1921): Diese Theorie begründet die Existenz der Unternehmung mit der These, daß aufgrund des Vorherrschens von Unsicherheit bestimmte nicht versicherbare Aktivitäten in einer Marktwirtschaft nicht durchgeführt werden; wird die Unsicherheit aber durch Unternehmer aufgefangen, so können diese Aktivitäten in Unternehmungen durchgeführt werden. Viele Ereignisse sind unsicher; kann man sich gegen diese versichern - Beispiele: Wasser- und Transportschäden -, so entsteht kein Problem. Ist jedoch der Ereignisraum nicht abgeschlossen oder unbekannt und sind die Wahrscheinlichkeiten für das Auftreten der unsicheren Ereignisse nicht zu ermitteln, so können sich die Produzenten gegen diese Ereignisse nicht versichern; als risikoaverse Produzenten werden sie die entsprechenden Aktivitäten unterlassen. Ereignisse, gegen die man sich versichern kann, bezeichnet Knight als Risiko, Ereignisse, gegen die man sich nicht versichern kann, als Unsicherheit. Bei Vorliegen von Unsicherheit werden demnach volkswirtschaftlich sinnvolle Aktivitäten von risikoaversen Produzenten unterlassen. 
Personen, die risikobewußt unsichere Aktivitäten durchführen, nennt Knight Unternehmer. Unternehmer sind Produzenten, die auch in einer unsicheren Welt produzieren. Die Aufgabe des Unternehmers ist es zum einen, die Nachfrage nach und das Angebot von Gütern abzuschätzen sowie die Rentabilität der verschiedenen Produktionsprozesse zu kalkulieren, und zum anderen, den gewählten Produktionsprozeß durchzuführen, d. h. zu lenken und zu kontrollieren. Besonders risikobewußte Personen gründen also Unternehmungen, um insbesondere unsichere Produktionsprozesse durchzuführen. Ihr Anreiz für diese Tätigkeit liegt in der Möglichkeit, einen Gewinn zu erzielen, ihre Strafe für das Eingehen zu waghalsiger Aktivitäten liegt in der Möglichkeit, einen Verlust zu erleiden; Gewinn und Verlust sind positive und negative Residualeinkommen, die keinem Produktionsfaktor zugerechnet werden können.

Wie Coase unterstellt auch Knight die Kontrolle der produktiven Tätigkeiten durch einen zentralen Entscheidungsträger. Für gewisse Aktivitäten sind die Funktionen der Übernahme von Unsicherheit und der Durchführung dieser Aktivitäten untrennbar. Der Gewinn ist quasi ein Anreiz, den die Gesellschaft setzt, diese Aktivitäten durchzuführen, obwohl sie nicht versicherbar sind; der Verlust ist entsprechend die Abschreckung, zu wagemutig und sorglos mit den gesellschaftlichen Ressourcen umzugehen.

Die Ausbeutungs-Theorie von Marglin (1974): Diese Theorie begründet die Existenz der Unternehmung mit der These, daß im Zuge eines evolutorischen Prozesses selbständiges Handwerk durch die Kapitalisten zerschlagen wurde, die damit den Produktionsprozeß unter ihre Kontrolle brachten und Arbeitsteilung und Arbeitszerlegung so bestimmten, daß sie einen möglichst großen Anteil am Sozialprodukt erhielten.

Zunächst bestand ein selbständiges Handwerk mit eigener Produktion und eigenen Absatzkanälen. Aufgrund steigender Skalenerträge im Absatz wurde es profitabel für Vermittler, den Transport und Absatz in größeren Nachfragezentren in eigener Regie zu übernehmen. Dadurch monopolisierten diese Vermittler die Informationen über den Absatzmarkt. Aufgrund dieser Zentralisierung des Absatzes und seiner Ablösung als eigenständige Funktion von der Produktion entstand eine Tendenz zur Auftragsarbeit; der Verlag entwickelte sich. Durch Zusammenfassung und Vervielfachung von Aktivitäten konnten steigende Skalenerträge in der Produktion ausgenutzt werden. Diejenigen, die eine große Anfangsausstattung besaßen, traten als Finanziers dieser Produktionen auf; die Kooperation und der Kapitalist entstanden. Die Janusköpfigkeit der Unternehmungsorganisation zeigte sich: Hierarchie als zentrale Leitung und als Herrschaft.

Aufgrund von technischen Entwicklungen und der Verringerung der Kosten der Durchsetzung und Kontrolle von Eigentumsrechten und Verhaltensbeschränkungen hinsichtlich der Aufteilung des Tages in Arbeitszeit und Freizeit und hinsichtlich der Arbeitsmethoden wurde eine verstärkte Arbeitsteilung und Arbeitszerlegung innerhalb der Unternehmungen profitabel; Arbeitsgesetze und Verordnungen sorgten für einen reibungslosen, leicht kontrollierbaren und hart sanktionierbaren Ablauf der Produktionen, einher ging eine Funktionalisierung und Hierarchisierung der Arbeiter; die Manufaktur entstand. Über Lerneffekte und technische Weiterentwicklungen sowie über weitergehende Durchsetzung von spezifischen Verfügungsbeschränkungen wurden 
Inventionen und Innovationen sowie die Zerlegung ganzheitlicher Tätigkeiten in genau definierte Aktivitäten rentabel; Entfremdungsprobleme traten auf. Arbeiter differenzierten sich nach Alter und Geschlecht; zusammen mit dem Auftreten von Disziplinierungsproblemen entstanden die Manager. Der Profit verblieb in der Unternehmung; die Fabrik entstand.

Machen wir uns noch einmal die Unterschiede in den Auffassungen der Unternehmungstheoretiker deutlich:

Nach Marglin erzwingt der Kontrolleur bei den Beschäftigten eine Arbeitszeit und Arbeitsmethoden, die ihren individuellen Interessen widersprechen. Bei Alchian und Demsetz ermöglicht der Kontrolleur es überhaupt erst, daß individuelle Rationalität der einzelnen Beschäftigten und kollektive Rationalität des Teams übereinstimmen, so daß das Einsetzen eines Kontrolleurs den individuellen Interessen jedes Beschäftigten entspricht. Wenn das nicht der Fall wäre, dann würden die Beschäftigten das Team verlassen.

Bei Coase und Knight wird von vornherein von-Über- und Unterordnungsverhältnissen in den Arbeitsbeziehungen ausgegangen, nur die Begründungen sind unterschiedlich. Während Coase ihre wesentliche Begründung in der - bis zu einer gewissen Ansammlung von abhängigen Arbeitnehmerverhältnissen gegebenen - Kostensenkung gegenüber alternativen Einzelproduktionen sieht (woraus umgekehrt abzuleiten ist, daß die Arbeiter ihrer Unterordnung in der Unternehmung solange zustimmen, wie sie als Einzelproduzenten nicht mehr verdienen können), besteht sie für Knight in der Verknüpfung des Tragens von Unsicherheit mit der Durchsetzung von erfolgversprechenden - also gewinnbringenden - Handlungen.

Gemeinsam ist allen ausgeführten Unternehmungstheorien die Ansicht, Unternehmungen beruhten auf Über- und Unterordnungsverhältnissen, auf Hierarchie, verschieden sind sie aber hinsichtlich deren Bedeutung für die Arbeitnehmer. Bei Alchian und Demsetz stimmen die Beschäftigten den Anordnungen der Vorgesetzten freiwillig zu, um die sonst unerreichbaren (Einkommens-)Vorteile der Teamproduktion zu erlangen, auch bei Coase haben die Arbeitnehmer ihre alternativen Verdienstmöglichkeiten als individuelle Einzelproduzenten im Auge, bei Knight tauschen risikoaverse Arbeitnehmer einen unsicheren Gewinn aus eigener Unternehmertätigkeit gegen einen relativ sicheren Lohn als untergebene Arbeitnehmer ein, und bei Marglin schließlich können die Arbeitnehmer aufgrund ihrer schwachen Verhandlungsposition nicht anders, als sich der Ausbeutung durch Kapitalisten zu unterwerfen.

\section{Arbeitsvertrag und Arbeitsorganisation}

Die vorgestellten Erklärungsansätze für die Existenz und Entwicklung von Unternehmungen enthalten als zentrales Theorieelement Aussagen über die Arbeitsorganisation. Deren elementarer Baustein ist der Arbeitsvertrag, mit dem wir uns nun näher befassen wollen. 
Alle marktmäßigen Tauschakte beruhen auf vertraglichen Übereinkünften der Tauschpartner, die oft - aber keineswegs immer - schriftlich fixiert werden. Im allgemeinen regelt der Vertrag alle Details: An einer nach Qualität und Quantität bestimmten Ware tritt der Verkäufer gegen einen bestimmten Preis seine Eigentumsrechte an den Käufer ab. Jede Vertragsseite spezifiziert ihren Beitrag zum Tausch in Leistung und Gegenleistung.

Nicht so beim Arbeitsvertrag. Wir heben zwei Aspekte der Besonderheit des Arbeitsvertrages hervor:

a) Fest umrissen ist hier nur die Leistung einer Vertragsseite, nämlich das vereinbarte Lohn- oder Gehaltentgelt. Demgegenüber ist für die Arbeitsleistung des Tauschobjekts "Arbeitskraft" lediglich ein Rahmen festgelegt; quantitativ etwa durch die Angabe der Arbeitszeit, qualitativ z. B. durch Berufs- oder Arbeitsplatzbezeichnung. Der konkrete Arbeitsinhalt ebenso wie die Arbeitsintensität bleiben in der Regel unbestimmt. Dies entspricht dem Interesse des Käufers, dem im Hinblick auf wechselnde Auftragslagen, technische Umstrukturierung der Kapitalgüter und Änderungen der Arbeitsorganisation daran gelegen ist, sich ohne Abschluß neuer Arbeitsverträge flexibel anpassen zu können.

b) Der Verkäufer, wiewohl er Eigentums- und Nutzungsrechte an seiner Arbeitskraft überträgt, bleibt dennoch als Person mit ihr verbunden, d. h. er verfügt letzten Endes weiterhin über das Verkaufte. Der Arbeitsvertrag bewirkt nicht die Eindeutigkeit der Neudefinition von Eigentumsrechten, wie sie aus anderen Tauschakten hervorgeht. Es entsteht vielmehr eine "Überlappung" der Dispositionssphären von Käufer und Verkäufer, die Anlaß für immer neue Konflikte ist.

Der Arbeitsvertrag enthält aufgrund seiner besonderen Form implizit auch eine Risikoaufteilung: Nach Knight entsteht die Unternehmung, weil für gewisse Risikoarten die Funktionen der Risikoübernahme und des Managements untrennbar sind. Danach trägt der Unternehmer das Verlustrisiko des in die Unternehmung eingebrachten Vermögens, die Gewinnchance ist hierfür der Anreiz. Dagegen bedeutet die Festlegung des Entgeltes im Arbeitsvertrag eine sichere Einkommenserwartung für den Arbeitnehmer. Die Nichtspezifizierung der Arbeitsleistung bewirkt eine andere Risikoaufteilung: Der Arbeitgeber hält sich die Möglichkeit der Einführung neuer Technologien und arbeitsorganisatorischer Maßnahmen offen, das Anpassungsrisiko geht weitgehend zu Lasten der Beschäftigten.

Beide zentralen Charakteristika von Arbeitsverträgen, sowohl die Unbestimmtheit der Arbeitsleistung als auch das mit jedem Arbeitsvertrag begründete Über- und Unterordnungsverhältnis, lassen sich auch in Begriffen von Kostensenkungen beschreiben und damit ökonomisch begründen. 
Für das Unbestimmtheitselement gilt:

Müßte in jedem Arbeitsvertrag auch die Arbeitsleistung genau spezifiziert werden, dann würde jede kleinste Veränderung des Arbeitsprozesses durch technische oder arbeitsorganisatorische Neuerungen den Abschluß neuer Arbeitsverträge erfordern, immense Kontraktkosten wären die Folge. Coase leitet als zentrale Begründung für die Entstehung von Unternehmungen her, daß die unternehmensinterne hierarchische Arbeitsorganisation Tausch(=Kontrakt)kosten, die bei Marktaktivitäten entstehen (Information, Verhandeln, etc.), senkt. Exakte Spezifizierung der Arbeitsleistung würde letztlich bedeuten, das Prinzip des Markttausches in die Unternehmung hineinzutragen, die damit ihren wesentlichen Existenzgrund verlieren würde.

Darüber hinaus reicht jeder Arbeitsvertrag in eine ungewisse Zukunft hinein; juristisch wird er ja auch als "Dauerschuldverhältnis" bezeichnet. Jeder übliche Vertrag enthält explizit oder implizit eine Klausel, in welchem Zustand der Welt er gelten soll oder nicht und was zu geschehen hat, falls er nicht erfüllt werden kann. Die Formulierung und das Verstehen von Zuständen der Welt, die erst in der Zukunft auftreten, ist jedoch oftmals zu komplex und daher ökonomisch unrentabel. Selbst wenn der Zustand der Welt exakt angegeben werden kann, treten möglicherweise Informationsprobleme über den tatsächlich eingetretenen Zustand auf; die Einigungskosten sind dann zu hoch.

Der Arbeitsvertrag behilft sich daher mit allgemeinen Formulierungen und überläßt alles Weitere dem "Direktionsrecht" des Arbeitgebers und der korrespondierenden "Weisungsunterworfenheit" des Arbeitnehmers. Damit kommen wir auch schon zum zweiten Charakteristikum von Arbeitsverträgen. Für das hierarchische Element in Arbeitsbeziehungen gilt (siehe dazu auch Arrow, K.J.):

Oft ist es kostengünstiger, Entscheidungen zentral zu treffen, als sie von getrennten Individuen herbeiführen zu lassen. Dafür sind folgende Gründe bedeutsam:

a) Eine optimale gemeinsame Entscheidung hängt von Informationen ab, die über die Individuen verstreut sind, kein Individuum allein kennt alle für die optimale Entscheidung wesentlichen Informationen. Eine Zusammenfassung von Individuen erhöht demnach die gemeinsame Information.

b) Die Zusammenfassung von Individuen zwecks Herbeiführung einer gemeinsamen Entscheidung verursacht Kosten in Form der Schaffung von Informationskanälen zwischen den Individuen. Hierarchische Informationskanäle sind aber aus den folgenden Gründen kostengünstiger als dezentral vermischte:

1) Die Transmission der Information zu einem zentralen Platz ist billiger als der gegenseitige Austausch.

2) Auf einen spezifischen Code - Fachsprache, Symbole - ausgelegte Informationskanäle filtern die Informationen und verringern damit die Übertragungs- und Verarbeitungskosten. 
3) Es ist billiger, wenn die Zentrale die kollektive Entscheidung trifft, als wenn alle Informationen wieder retransmittiert werden.

Oft ist es kostengünstiger, Handlungen nicht dezentral über Märkte, sondern über einen hierarchischen Organisationsmechanismus zu koordinieren. Dafür sind die folgenden Gründe wesentlich:

a) Die Formulierung eines Vertrages derart, daß einer Autorität prinzipiell zu gehorchen ist, reduziert die Tansaktionskosten, senkt die Kosten der Unsicherheit und stabilisiert die wechselseitigen Erwartungen im Vergleich zu einer dezentralen Marktorganisation. Die Autorität kann dabei eine Person beziehungsweise deren Position oder ein Normensystem sein.

b) Die Transaktions- und Unsicherheitskosten sinken darüber hinaus durch

- Erzeugung von Loyalität und wechselseitigem Vertrauen

- Installierung von Aufstiegsleitern und Senioritätsregeln

- Bildung von Spezialwissen und -fähigkeiten, die andernorts nur schwer verwertbar sind.

In allen drei Fällen steigen die Abwanderungskosten aus dem Arbeitsvertrag.

c) Die Informations- und Entscheidungskosten sinken darüber hinaus durch

- Formalisierung von Positionen und Rollen

- Abgrenzung der Entscheidungskompetenzen

- Arbeits- und Aufgabenspezialisierung.

In allen drei Fällen entsteht ein Mixtum aus persönlicher Autorität - via Person, Position oder Rolle - und aus unpersönlicher Autorität - via Normen, Regeln und Vorschriften.

Wir halten fest, daß Produktion dominant in Unternehmungen organisiert wird, die sich ausnahmslos hierarchischer Organisationsverfahren bedienen. Zwar operieren Unternehmungen auf Beschaffungs- und Absatzmärkten und unterhalten Außenbeziehungen mit Marktumwelten, sie koordinieren aber ihre internen Produktionstätigkeiten gerade nicht markimäßig, sondern hierarchisch.

\section{Begründung für Mitbestimmungsrechte der Arbeitnehmer}

Eine andere, nicht weniger wichtige Frage ist dann, inwieweit der Hierarchiemechanismus Legitimität beanspruchen kann, d.h. auf freiwilliger Zustimmung der ihm unterworfenen Arbeitskräfte beruht. Diese Frage läßt sich in abstrakten ökonomischen Begriffen recht einfach beantworten: Eine hierarchische Arbeitsbeziehung gestattet in dem Maße die Ausübung von Zwang, wie die Abwanderung aus dem Arbeitsvertrag dem Weisungsunterworfenen nicht ohne Nutzeneinbußen möglich ist. Diese Fest- 
stellung soll abschließend erläutert werden. Eine klare Formulierung verdanken wir K. J. Arrow (S. 63 f.):

"Staat und Unternehmungen gleichen sich darin, daß sie eine ... Art von autoritativer Kontrolle über ihre Beschäftigten ausüben. Tatsächlich ist der Arbeitsvertrag, wie Herbert Simon betont hat, exakt ein Vertrag, aufgrund dessen der Beschäftigte in Weisungsunterworfenheit einwilligt. Dieser Vertrag unterscheidet sich von einem Güter-Kaufvertrag; was gekauft und verkauft wird, ist keine eindeutige, objektive Sache, sondern eher eine persönliche Beziehung. Innerhalb des Vertragsrahmens ist die Beziehung zwischen Beschäftiger und Beschäftigtem keine Marktbeziehung mehr, sondern eine Autoritäts-Beziehung. Natürlich ist das Ausmaß dieser Autorität üblicherweise durch die Vertragsmodalitäten und, grundlegender, durch die Freiheit des Beschäftigten begrenzt, sein Arbeitsverhältnis zu kündigen. Das Autoritätsausmaß ist aber nicht unbedeutend, weil die Ausübung dieser Freiheit normalerweise mit einigen Kosten verbunden ist."

Da jeder Beschäftigte einer Unternehmung das Recht hat zu kündigen, könnte man in Anlehnung an Alchian und Demsetz die Existenz von besonderen Autoritätsbeziehungen - i. S. von potentieller Zwangsausübung - innerhalb von Unternehmungen leugnen; d. h. ein Beschäftigter, der nicht kündigt, akzeptiert damit unausgesprochen die Weisungen seiner Vorgesetzten, er schließt permanent implizite Verträge über die Art seiner Tätigkeit im Unternehmen. Eine solche Hypothese gilt jedoch nur unter der Voraussetzung, daß die (monetären und psychischen) Kosten eines Arbeitsplatzwechsels für den Beschäftigten gleich Null oder zumindest vernachlässigbar gering sind. Arrow weist nun jedoch zu Recht darauf hin, daß in der Realität ein Arbeitsplatzwechsel für den Beschäftigten mit positiven Abwanderungskosten verbunden ist. Folgende Kosten gehören dazu: Verlust an informellen Beziehungen mit befreundeten Arbeitskollegen; Verlust an Aufstiegsmöglichkeiten in der Unternehmung; Entwertung des Humanvermögens hinsichtlich betriebsspezifischer Qualifikationen; häufig auch Kosten durch Umzug und in diesem Zusammenhang auch Verlust nachbarschaftlicher Beziehungen, Schulwechsel der Kinder etc.; Suchkosten, eine neue Beschäftigung zu finden; Kosten, am neuen Arbeitsplatz und am neuen Wohnort neue soziale Beziehungen aufzubauen.

Demgegenüber sind die Kosten des Arbeitgebers zu berücksichtigen, einen freigewordenen Arbeitsplatz neu zu besetzen. Sie sind um so höher, je schwieriger es ist, Arbeitskräfte mit der geforderten Qualifikation über den Arbeitsmarkt zu bekommen (Suchkosten), und je mehr der Arbeitgeber durch Trainee-Programme, Einarbeitungszeit etc. für die entsprechenden Arbeitskräfte an Investitionen in Humanvermögen aufwenden muß. In der Literatur wird häufig davon ausgegangen, daß die Kosten des Arbeitgebers vergleichsweise gering sind. (Vgl. zu diesen Problemen auch Nutzinger, H.G.: Firm, sowie Brandes, W./Weise, P.).

Aus alledem folgt: Je höher die Kosten eines Beschäftigten, seinen Arbeitsplatz zu wechseln (= Kosten der Abwanderung), und je niedriger die Kosten des Arbeitgebers, den frei gewordenen Arbeitsplatz neu zu besetzen, in desto stärkerem Maße wird ceteris paribus die Vertragsbeziehung zwischen Beschäftigtem und Arbeitgeber durch eine Autoritätsbeziehung mit Zwangselementen verdrängt. 
Damit wird klar, daß das Modell der Unternehmung von Alchian und Demsetz nur dann ein relativ genaues Abbild einer realen Unternehmung liefert, wenn die Kosten der Abwanderung für die Beschäftigten relativ gering sind; das dürfte aber für die Mehrzahl der Beschäftigten in realen Unternehmen nicht zutreffen. Die Unternehmungsmodelle von Coase und Knight sind in diesem Punkt wesentlich realistischer. Marglin hingegen geht von prohibitiv hohen Kosten der Abwanderung aus, bei ihm haben die Arbeiter keine Alternative zur Ausbeutung durch die Kapitalisten. Die verschiedenen referierten Unternehmungstheorien liegen daher auf einem Kontinuum bezüglich der (jeweils nur implizit vorausgesetzten) Abwanderungskosten aus dem Arbeitsvertrag.

Allgemein wird also die Existenz der Unternehmung mit der These begründet, daß eine geeignet miteinander verschränkte Kombination von individuellen Produktionshandlungen kostengünstiger ist als ein über Marktbeziehungen koordinierter Produktionsprozeß. Insbesondere gilt dies, wenn der Produktionsprozeß so auf Elementarprozesse aufgeteilt ist, daß Arbeitsteilung und -spezialisierung optimal durchgeführt werden kann und daß die Leerzeiten möglichst gering werden. ${ }^{12}$ Voraussetzung für den Kostenvorteil der hierarchischen Organisation ist allerdings die Sicherheit, daß jedes Individuum seine zugewiesenen Aufgaben erfüllt. Diese Sicherheit wiederum ist nur zu erreichen, wenn die Kosten der Abwanderung aus einem Arbeitszusammenhang für die Arbeitskräfte erheblich sind. Die (fast) kostenlosen Abwanderungsmöglichkeiten bei einem Marktmechanismus werden ersetzt durch hohe Austrittskosten bei einer hierarchischen Organisationsform.

Sind die Abwanderungskosten hoch, haben die Beschäftigten einen Anreiz, ihre Interessen durch die Strategie Widerspruch durchzusetzen. ${ }^{13}$ Sie werden von dieser Strategie umso häufiger Gebrauch machen, je höher die Kosten der Abwanderung und je geringer die Kosten des Widerspruchs sind (d. h. die Kosten der Organisation des Widerspruchs sowie die Kosten durch die erwarteten Sanktionen des Arbeitgebers). Tatsächlich läßt sich die Geschichte der industriellen Beziehungen und des Arbeitsrechts als schrittweise Institutionalisierung von drei zentralen "Widerspruchsmechanismen" interpretieren:

a) Etablierung von Gegenmacht durch Gewerkschaften und Streikdrohung;

b) Durchsetzung von Arbeitsrecht als Arbeitnehmerschutzrecht;

c) Einführung von Demokratieelementen im Arbeitsverhältnis und in der Unternehmung durch Mitbestimmungs- und Betriebsverfassungsrecht.

In abstrakten Begriffen läßt sich auch für die Widerspruchsstrategie das Ausmaß von Zwangselementen im Arbeitsverhältnis umreißen: Je höher die Kosten des Widerspruchs für die Beschäftigten, desto stärker sind ceteris paribus die Autoritätsbeziehungen in der Unternehmung (und umgekehrt). 


\section{C. Ökonomische Analyse der Mitbestimmung}

\section{Spezifische Ressourcen, Organisationsrente und Mitbestimmung}

Wir wollen nun die Erkenntnisse des vorangehenden Kapitels verallgemeinern. Eine Unternehmung ist letztlich dadurch gekennzeichnet, daß verschiedene Eigentümer knapper Ressourcen sich zusammentun, um gemeinsam bestimmte Güter und Dienstleistungen zu produzieren, indem sie sich eine - wie unter 2. ausgeführt - spezifische Organisationsform geben. Wesentlich für das Verständnis der Koordination von Entscheidungen bezüglich der Nutzung dieser Ressourcen ist die Tatsache, daß zumindest für einige dieser Ressourcen gilt: Der Wert der Ressource in der nächstbesten Verwendung außerhalb der Unternehmung ist geringer als der Wert dieser Ressource bei gemeinsamer Nutzung in der Unternehmung. Die Höhe dieser Differenz mißt den Grad der Spezifität einer Ressource (Alchian, A.A., ZgS; Williamson, O.E.), die Kosten der Abwanderung (Weise, P. et al.) bzw. das konkrete Vertragsinteresse des entsprechenden Ressourceneigentümers (Stützel, W.). Aus der Sicht aller Eigentümer unternehmensspezifischer Ressourcen zusammen besteht somit eine "Organisationsrente" (Aoki, M.: Firm) oder "Quasirente" (Alchian, A.A., ZgS), die durch Kooperation erwirtschaftet werden kann und über deren Verteilung man sich einigen muß. ${ }^{14}$ Das Recht, Entscheidungen über die Nutzung der in die Unternehmung eingebrachten Ressourcen zu fallen, erhäl somit für alle Eigentümer unternehmensspezifischer Ressourcen einen Wert. ${ }^{15}$ Andererseits besteht in der Regel für den Inhaber von Entscheidungsrechten ein Anreiz, einen Teil dieser Rechte an Spezialisten zu delegieren und sich auf die Einstellung, Kontrolle und Entlassung dieser Spezialisten zu beschränken; dadurch können Transaktionskosten gesenkt und Spezialisierungsvorteile genutzt werden.

Historisch hat sich während des Industrialisierungsprozesses die "kapitalistische Unternehmung" durchgesetzt, die dadurch gekennzeichnet ist, daß alle Entscheidungsund Kontrollrechte ausschließlich bei den Kapitaleignern liegen; den Eignern der Ressource Arbeit werden keine Entscheidungs- und Kontrollrechte eingeräumt. Für diese Entwicklung lassen sich zwei Gründe anführen. Zum einen ist Kapital in quantitativer, qualitativer, räumlicher und zeitlicher Hinsicht wesentlich anpassungsfähiger als Arbeit (vgl. Brandes, W./Weise, P.; siehe auch Putterman, L., EI):

- Kapital kann sich hinsichtlich der Quantität relativ schnell anpassen: Kapitalgüter werden in größerer oder geringerer Menge produziert. Die Produktion von Arbeitskräften ist dagegen viel langsamer und nur lose an ökonomischen Kriterien orientiert. Kapital hat bezüglich der Quantität also einen Anpassungsvorteil. D. C., QREB und von Brandes, W. et al. JNPÖ. 
- Kapital, verkörpert in Kapitalgütern, ist nur bedingt verformbar; doch wird es über Abschreibungen wieder liquide und kann sich in anderen Güterqualitäten konkretisieren. Arbeit dagegen ist hinsichtlich der Qualitat, vor allem bei schwierigeren Tätigkeiten, nur mit großen Kosten veränderbar (Umschulung usw.). Kapital hat demnach bezüglich der Qualität einen großen Anpassungsvorteil.

- Kapital kann ohne Mühe im Raum bewegt werden. Die Mobilität der Arbeit dagegen unterliegt großen Hemmnissen und ist mit hohen Kosten verbunden, da der Arbeiter als Person sich im Raum bewegen muß: Aufgeben der sozialen Umgebung, Umzugskosten usw. Kapital ist bezüglich des Raumes also weitaus anpassungsfähiger als Arbeit.

- Dadurch, daß Kapital immer wieder liquide wird und sich in anderen Formen konkretisieren kann, bleibt es sozusagen immer jung; es wird nicht unproduktiver und unterliegt keinem Verschleiß. Demgegenüber wird der Arbeiter immer älter und ab einer gewissen Grenze auch unproduktiver; den Jungbrunnen gibt es nur im Märchen. Bezüglich der Zeit ist Kapital also erheblich anpassungsfähiger als Arbeit.

Zum anderen war die Masse der Arbeiter besitzlos. Der typische Beschäftigte verfügte somit über keine Sicherheiten, um am Kapitalmarkt in nennenswertem Umfang Kredite aufnehmen und eine eigene Unternehmung gründen zu können (s. auch Eger, T./Weise, P.: Evolution, und Vogt, W.).

Die Arbeiter hatten somit eine erheblich schwächere Verhandlungsposition als die Kapitaleigner und standen vor der Alternative, entweder zu den von den Kapitaleignern gesetzten Bedingungen zu arbeiten oder überhaupt nicht zu arbeiten und zu verhungern. Aufgrund der Zerlegung der Produktionsprozesse in eine Vielzahl leicht zu bewältigender Teilaufgaben und aufgrund der Koordination dieser Teilaufgaben durch Autoritätsbeziehungen blieben die Arbeiter zum größten Teil leicht ersetzbar; die Bildung von unternehmensspezifischem Humankapital spielte eine relativ geringe Rolle (siehe auch Edwards, R.). Aufgrund dieser sehr schwachen Verhandlungsposition der Arbeiter sicherten sich die Kapitaleigner die exklusiven Entscheidungs- und Kontrollrechte.

Heute hat sich demgegenüber die Situation zumindest in zweierlei Hinsicht drastisch verändert: Zum einen ist die Verhandlungsposition der Arbeiter durch kollektive Interessenvertretung seitens der Gewerkschaften, soziale Sicherung, Arbeitsschutzgesetzgebung u.a.m. stark verbessert worden; zum anderen ist die Bedeutung der Ausbildung der Arbeitskräfte im allgemeinen und die Bedeutung unternehmensspezifischer Qualifikationen im besonderen stark gestiegen. Schließlich hat der Staat ein Interesse an einer möglichst konfliktfrei durchgeführten Produktion. Es ist somit zu überprüfen, ob Mitbestimmung auf Unternehmensebene ein geeigneter Mechanismus sein könnte, die Konflikte zwischen Kapital und Arbeit um die Verteilung der Organisationsrente sowie die Konflikte zwischen "gewöhnlichen" Beschäftigten und Management um die Gestaltung der Arbeitsbedingungen effizient zu regeln. 


\section{Konflikte zwischen Kapital und Arbeit}

Haben die Beschäftigten trotz positiver Abwanderungskosten keine Mitbestimmungsrechte, so kann das die folgenden ökonomisch negativen Auswirkungen haben. Zum einen besteht ein verminderter Anreiz, in unternehmensspezifisches Humankapital zu investieren (z. B. durch Lohnverzicht während der Ausbildungszeit), wenn man nicht sicher sein kann, daß diesen Investitionen in Zukunft auch angemessene Erträge gegenüberstehen. ${ }^{16}$ Zum anderen besteht ein verstärkter Anreiz, sich durch kostspielige spontane Aktionen gegen Managemententscheidungen zur Wehr zu setzen, die zu einer Entwertung unternehmensspezifischen Humankapitals führen. Durch Mitbestimmung auf Unternehmensebene könnten diese Anreizprobleme gemildert werden. Dabei treten allerdings zwei Probleme auf. Einerseits schwächen Mitbestimmungsrechte der Arbeitnehmer die Verfügungsrechte der Kapitaleigner. Der Anreiz, Realkapital für mitbestimmte Unternehmen bereitzustellen, sinkt, da die Anteilseigner nicht mehr sicher sein können, daß alle renditesteigernden Maßnahmen die Zustimmung der Arbeitnehmervertreter finden. ${ }^{17}$ Andererseits wird bei einer gesetzlich verordneten Mitbestimmung nicht berücksichtigt, daß der Umfang unternehmensspezifischer Investitionen in Humankapital bei den einzelnen Beschäftigten sehr unterschiedlich ist. Es ist somit möglich, daß die mobileren Arbeitnehmer ihre Mitbestimmungsrechte dazu nutzen, die weniger mobilen Arbeitnehmer und die Kapitaleigner auszubeuten. ${ }^{18}$

Reichen diese Probleme hin, eine gesetzlich verordnete Mitbestimmung als ökonomisch ineffizient zu verwerfen? Eine endgültige ökonomische Würdigung müßte zumindest noch die folgenden Gesichtspunkte berücksichtigen: Zum einen läßt es sich zwar nicht von der Hand weisen, daß durch die Einführung der Mitbestimmung bestimmte Investitionen, die Arbeitsplätze vernichten und Arbeitsbedingungen verschlechtern, nicht mehr durchgesetzt werden können, obwohl sie vor Einführung der Mitbestimmung mit einer hohen Rendite für die Kapitaleigner verbunden waren. Aber sofern die Investitionen zu einer Externalisierung von Risiken auf die relativ immobilen Arbeitnehmer führten, verzerrten die Renditen den tatsächlichen Wert der Investitionen für die Gesellschaft. Im günstigsten Fall führt Mitbestimmung zu einer Beseitigung dieser Externalisierungsmöglichkeiten, im weniger günstigen Fall führt Mitbestimmung zu einer Externalisierung von Kosten seitens der Beschäftigten auf die Kapitaleigner und somit zu einer Verzerrung der Renditen in die andere Richtung. Zum anderen ist

Hierbei ist die relative Aufteilung der Ausbildungskosten auf Unternehmung und Beschäftigte $\mathrm{zu}$ beachten. Zu einer theoretischen Analyse dieser Aufteilung der Ausbildungskosten siehe Kraft, K.: Partizipation. Zu einer empirischen Untersuchung der Bedeutung unternehmensspezifischer Qualifikationen siehe Biehler, H. et al.

17 Diesen Gesichtspunkt betonen Kritiker des Mitbestimmungsgesetzes. Vgl. etwa Pejovich, S. und Prosi, G.

Furubotn schlägt deshalb vor, den einzelnen Beschäftigten Anteilsrechte für die von ihnen realisierten unternehmensspezifischen Humankapitalinvestitionen zu gewähren, die nicht übertragbar sind und für die Dauer der Beschäftigung im Unternehmen gültig sind. Stimmrechte und Anteile am ausgeschütteten Gewinn bemessen sich nach der relativen Höhe dieser Anteile. Vgl. Furubotn, E., JoB, ders.: General Model. 
zu analysieren, ob Mitbestimmung die Qualität der Managemententscheidungen und damit die "interne Effizienz" der Unternehmung beeinflussen könnte. Mit diesem zweiten Gesichtspunkt wollen wir uns im folgenden Abschnitt etwas ausführlicher befassen.

\section{Konflikte zwischen Beschäftigten und Management}

Wie bereits unter B.II. erwähnt, sind Arbeitsverträge nicht vollständig spezifiziert, d. h. Arbeitsverträge enthalten eine beträchtliche Anzahl "freier Variablen". Diese freien Variablen werden zu einem großen Teil vom Management kontrolliert, dessen Weisungsbefugnis sich die Beschäftigten innerhalb der vertraglich festgelegten Grenzen unterwerfen. Da aber eine vollständige Kontrolle aller relevanten Dimensionen des Arbeitsverhaltens der Beschäftigten durch das Management mit prohibitiven Kosten verbunden ist, unterliegt ein Teil der freien Variablen tatsächlich der Kontrolle der Beschäftigten. Die vom Management kontrollierten Variablen wollen wir im folgenden kurz als "Arbeitsbedingungen" bezeichnen. Darunter fallen beispielsweise Ausgestaltung und Sicherheit der Arbeitsplätze, Arbeitsanforderungen, Flexibilität der Arbeitszeit, Ausmaß hierarchischer Kontrollen, innerbetriebliche Weiterbildung usw. Die (zumindest teilweise) von den Beschäftigten kontrollierten Variablen könnte man unter dem Stichwort "Arbeitsqualität" zusammenfassen. Hierunter fallen etwa die Arbeitsintensität, die Präzision bei der Herstellung von Gütern und Dienstleistungen, die Sparsamkeit beim Verbrauch von Material und Energie sowie die Sorgfalt bei der Nutzung von Maschinen und Werkzeugen. ${ }^{19}$

Da im Zuge der Zeit sowohl die Freizeit als auch das Realeinkommen der Arbeiter gestiegen sind und da in den industrialisierten Ländern in allen Lebensbereichen demokratische Organisationsformen autoritäre Entscheidungsprozesse teilweise verdrängt haben, sind die Arbeiter nicht mehr bereit, in einer Unternehmung ausschließlich durch Befehl und ohne jegliche Entscheidungs- und Kontrollrechte zur Arbeit angehalten $\mathrm{zu}$ werden. Sie reagieren auf autoritäre Organisationsformen mit einer geringeren Leistungsintensität und höheren Absenzzeiten..$^{20}$ Mitbestimmung könnte ein Vehikel dafür sein, die Arbeitsmotivation in einer Unternehmung bei erhöhter Demokratisierung einer Gesellschaft zumindest nicht sinken zu lassen. Diesem Vorteil steht der Nachteil einer aufwendigeren Informationsverarbeitung und Entscheidungsfindung gegenüber.

Die Bedeutung der Arbeitsmotivation für die Produktivität wird in letzter Zeit verstärkt auch von der theoretischen Literatur über Arbeitsmarktprozesse erkannt; vgl. hierzu beispielsweise MacLeod, W.B. Für die Betrachtung der Arbeitsintensität aus juristischer Perspektive siehe Dorndorf, E., ZfA.

20 Die Diskussion um Führungsstile, das japanische Modell, Tätigkeitserweiterungen und Gruppenarbeit bestätigt diese Aussage. Empirische Befunde und theoretische Analysen zur Arbeitsmotivation finden sich bei Graumann, C.F.; zu Informations-, Anreiz- und Verhandlungsstrukturen in der japanischen Wirtschaft vgl. Aoki, M.: Information. 
Sofern für die Beschäftigten positive Abwanderungskosten bestehen, ist das Management in der Lage, sich bei der Festlegung der "Arbeitsbedingungen" wie ein Monopolist zu verhalten. Wenn wir in einem ersten Schritt einmal annehmen, daß das (nichtmitbestimmte) Management die Interessen der Kapitaleigner zu seinen eigenen gemacht hat, wird es die Arbeitsbedingungen so festlegen, daß der Profit unter Berücksichtigung der erwarteten Anpassung der Beschäftigten in ihrer Arbeitsqualität an die jeweils gesetzten Arbeitsbedingungen maximal ist. Es läßt sich nun zeigen, daß unter den üblichen Annahmen der resultierende Zustand - wie jedes Monopolgleichgewicht ineffizient ist: Die Grenzrate der Substitution im Konsum zwischen Arbeitsbedingungen und Arbeitsqualität entspricht nicht der entsprechenden Grenzrate der Substitution in der Produktion, und die Summe der individuellen Grenzraten der Substitution zwischen Arbeitsbedingungen (Arbeitsqualität) und Einkommen entspricht nicht der Grenzproduktivität der Arbeitsbedingungen (Arbeitsqualität). Arbeitsbedingungen und Arbeitsqualität sind schlechter als bei effizienter Allokation, beide Parteien könnten durch einen Austausch guter Arbeitsbedingungen gegen gute Arbeitsqualität gewinnen. ${ }^{21}$

Mitbestimmung könnte einen effizienten Trade-off zwischen Arbeitsbedingungen und Arbeitsqualität erleichtern, indem dem Management mehr Informationen über wechselseitige Verbesserungsmöglichkeiten zur Verfügung gestellt werden und indem die Beschäftigten ein stärkeres Vertrauen in Zusagen des Managements haben, daß kooperatives Verhalten nicht ausgebeutet wird. ${ }^{22}$

In der Realität kann man aber nicht notwendig davon ausgehen, daß die Ziele der Manager mit denen der Kapitaleigner identisch sind. Manager verfolgen auch eigene Ziele. Sie fragen Status, Prestige, Bequemlichkeit u.a.m. nach und sind bemüht und in der Lage, Informationen zu monopolisieren, um die eigenen Erfolge zu übertreiben und die Erfolge anderer zu untertreiben. ${ }^{23}$ Das Verhalten von Managern auf allen Hierarchiestufen hat somit gewisse Ähnlichkeiten mit dem Verhalten von Bürokraten in öffentlichen Verwaltungen (vgl. hierzu etwa Downs, A., AER). Die Bürokratie großer Wirtschaftsunternehmen unterscheidet sich allerdings in zwei wesentlichen Punkten von der Bürokratie öffentlicher Verwaltungen. Zum einen wird der Output von Wirtschaftsunternehmen mit Marktpreisen bewertet, der Output von öffentlichen Verwaltungen dagegen nicht. Das hat zur Folge, daß sich der Erfolg einer Unternehmung leichter messen läßt als der Erfolg einer Behörde. Zum anderen können die Anteilseigner einer privatwirtschaftlichen Unternehmung ihre Anteile veräußern, wenn

$21 \mathrm{Zu}$ diesen und ähnlichen Argumenten vgl. McCain, R.A., ZfN, ders.: Codetermination; Leibenstein, H., AER 1982, AER 1983 u. ders.: Firm. trust"-Situationen im Unternehmen siehe die bahnbrechende Monographie von A. Fox. Die teilweise Substitution von Kontrolltechniken durch Aufbau von Vertrauensbeziehungen in Unternehmen ist Bestandteil fast aller neuen Management- und Führungstechniken und der darauf bezogenen Managementtheorien. 
sie mit dem Erfolg der Unternehmung nicht zufrieden sind. Werden die Anteile an der Börse gehandelt (Aktien), dann reflektiert deren Kurs somit - mit gewissen Einschränkungen - die Bewertung der wirtschaftlichen Tätigkeit der Unternehmung durch die Anbieter von und die Nachfrager nach Anteilsrechten. Die "Eigentümer" einer öffentlichen Verwaltung, die Steuerzahler, haben demgegenüber nicht die Möglichkeit, ihre Anteile zu veräußern - es sei denn, sie wandern aus.

Der Kapitalmarkt und die Konkurrenz der Manager untereinander beschränken somit die Möglichkeit der Manager, ohne Sanktionen eigene Ziele zu Lasten der Interessen der Anteilseigner zu verfolgen. Aber auch diese Mechanismen sind nur sehr unvollkommen in der Lage, die Qualität der Managerleistungen zu offenbaren - zumal Manager in der Regel vergleichsweise mobil sind und zum Teil eine erhebliche Zeitverzögerung zwischen Managemententscheidungen und deren Auswirkungen auf den wirtschaftlichen Erfolg der Unternehmung besteht. ${ }^{24}$

Durch Mitbestimmung wird ein zusätzlicher Mechanismus zur Kontrolle von Managemententscheidungen installiert. ${ }^{25}$ Der mitbestimmte Aufsichtsrat verfügt über mehr Informationen, die die Qualität des Managerverhaltens betreffen, als ein Aufsichtsrat, der ausschließlich die Interessen der Kapitaleigner repräsentiert. Ob durch diese zusätzliche Managementkontrolle die Anreize der Manager zu ineffizientem Verhalten gemildert werden oder ob sich Manager und sonstige Beschäftigte zu Lasten der Kapitaleigner arrangieren, ist eine empirische Frage. Ein theoretisches Argument für eine effizientere Managementkontrolle durch Mitbestimmung besteht darin, daß der Zeithorizont der vergleichsweise mobilen und vergleichsweise alten Manager kürzer ist als der Zeithorizont der vergleichsweise immobilen und vergleichsweise jungen sonstigen Beschäftigten (Smith 1991). Mitbestimmung könnte dann dazu beitragen, die Manager darin zu hindern, kurzfristige Erfolge zu Lasten der langfristigen Steigerung des Unternehmenswerts anzustreben.

\section{Spontane Entwicklung versus gesetzliche Verordnung von Mitbestimmung}

Die ökonomische Analyse der Mitbestimmung hat einige Argumente hervorgebracht, die für eine Mitbestimmung sprechen, sie hat aber zu keinem eindeutigen Ergebnis geführt. Mitbestimmung schafft einerseits Anreize, spezifisches Humankapital bereitzustellen, vermindert aber andererseits den Anreiz, Realkapital bereitzustellen. Mitbestimmung führt dazu, ein Absinken der Arbeitsmotivation in einer demokratischen Umwelt zu verhindern; der Preis hierfür ist aber der Zeitbedarf für Informationsverarbeitung und Entscheidungsfindung. Mitbestimmung schafft zwar Bedingungen dafür, einen effizienten Trade-off zwischen Arbeitsbedingungen und Arbeitsqualität zu erreichen; aber werden diese Bedingungen tatsächlich genutzt? Mitbestimmung schafft Smith, S.C., JEBO. 
zwar die Möglichkeit, durch interne Kontrolle ineffizientes Managementverhalten abzubauen; aber wird diese Möglichkeit auch genutzt? Darüber hinaus senkt Mitbestimmung die Kosten der Durchsetzung von Entscheidungen, wenn einmal Konsens erzielt ist; sie verursacht allerdings höhere Entscheidungsfindungskosten, um überhaupt erst einen Konsens zu erzielen.

Überwiegen nun die positiven oder die negativen ökonomischen Konsequenzen der Mitbestimmung? Ist Mitbestimmung im engeren ökonomischen Sinne effizient? Einige Gegner einer gesetzlich verordneten Mitbestimmung vertreten die Auffassung, daß diese Frage bereits "durch den Markt" beantwortet wurde. Wäre nämlich die Mitbestimmung effizient, so hätten die Unternehmen sie freiwillig eingeführt. Da sie das nicht getan haben, sondern vom Gesetzgeber dazu gezwungen werden mußten, kann sie nicht effizient sein (vgl. etwa Pejovich, S.; Jensen, M./Meckling, W., JoB). Aber der Markttest ist kein hinreichender Beweis dafür, daß Mitbestimmung ineffizient ist. Es ist vielmehr zu vermuten, daß sich die mitbestimmte Unternehmensverfassung auch dann nicht spontan, durch freiwillige Verträge, durchsetzt, wenn sie effizient ist. Das hängt damit zusammen, daß niemand freiwillig auf Verfügungsrechte verzichtet, wenn ungewiß ist, ob er jemals eine Entschädigung dafür erhält. Die Manager haben durch die Einführung von Mitbestimmung zunächst einmal einen definitiven Nachteil: Sie müssen zwei Herren dienen, und sie sind zusätzlichen Kontrollen ausgesetzt; die Managementtätigkeit wird mühseliger. Selbst wenn Produktivitätsvorteile der Mitbestimmung mit hinreichender Sicherheit erwartet werden könnten, bestünde für die Manager das Problem, sich einen hinreichend großen Teil des Produktivitätszuwachses als Entschädigung für die erlittenen Nachteile zu sichern. Es ist aber sehr zweifelhaft, ob die Beschäftigten und die Anteilseigner auf Dauer bereit sein werden, die Manager für den Verlust an Privilegien zu entschädigen. ${ }^{26}$

Die Manager haben also auch dann, wenn Mitbestimmung effizient ist, nur einen geringen Anreiz, sich gegenüber den Anteilseignern für eine mitbestimmte Unternehmensverfassung stark zu machen. Aber haben nicht die Anteilseigner ein Interesse daran, auch gegen den Widerstand des Managements effiziente Organisationsformen durchzusetzen? Die Anteilseigner haben nur insofern ein Interesse an einer effizienten Organisationsform, als dadurch ihre Rendite positiv beeinflußt wird. Wenn Mitbestimmung effizient ist, ist es zwar grundsätzlich möglich, den Anteilseignern eine höhere Rendite zu sichern, ohne die anderen Unternehmensmitglieder schlechter zu stellen. Es ist aber nicht sicher, ob die (mitbestimmungsberechtigten) Beschäftigten dem auch zustimmen. Es gibt aber auch noch ein grundsätzliches Argument dafür, daß sich renditeorientierte Anteilseigner einer freiwilligen Einführung der Mitbestimmung auch 
dann widersetzen werden, wenn sie effizient ist. Dies resultiert daraus, daß die Einführung von Mitbestimmungsrechten ihre effizienzsteigernden Wirkungen erst dann entfaltet, wenn die Beschäftigten spezifische Qualifikationen aufgebaut haben und wenn das Management die erforderlichen neuen Führungstechniken erlernt hat. "Klassisch"-kapitalistische Eigentumsrechte, nicht-spezifische, mobile Arbeitskräfte und rigide hierarchische Kontrollen können sich daher über längere Zeiträume wechselseitig stabilisieren, auch wenn effizientere Zustände mit Mitbestimmungsrechten, spezifisch-qualifizierten, immobilen Arbeitskräften und partizipativem Führungsstil realisierbar wären. ${ }^{27}$

Hinzu kommen einige weitere Argumente, die gegen eine spontane "freiwillige" Einführung der Mitbestimmung - ja, gegen jede spontane Entwicklung einer gesellschaftlichen Regel - , obwohl sie effizient ist, sprechen. Ein bösartiges Argument zuerst: Fast alle sozialen und wirtschaftlichen Errungenschaften, die letztendlich der Verbesserung der Funktionsfähigkeit der Wirtschaftsgesellschaft dienten, wurden gegen den erbitterten Widerstand von Kapitaleignern und Managern eingeführt, wie beispielsweise der Übergang von der 78- zur 72-Stunden-Woche, Mindeststandards für Arbeitsbedingungen oder Umweltschutzauflagen. Ein zweites Argument: Der Staat steht nicht exogen außerhalb des Wirtschaftsprozesses, sondern ist endogen selbst ein Teil des gesellschaftlichen Evolutionsprozesses. So wurden beispielsweise die Vorläufer der heutigen Arbeitsgesetze, die autoritären Gesindeordnungen von 1810 und 1854, auf Druck der Unternehmer erlassen und hatten selbstverständlich einen über das Privatrecht hinausgehenden öffentlichen Charakter. Die Gegenüberstellung einer (effizienten) spontanen Entwicklung der (ineffizienten) gesetzlichen Verordnung ist mithin theoretisch nicht haltbar. Ein drittes Argument: Die unqualifizierte Aussage, eine effiziente Regel setze sich spontan in der Evolution durch und eine gesetzliche Verordnung erweise sich qua Umkehrschluß als ineffizient, ist aus theoretischen Gründen eindeutig falsch: Der Zufall kann entscheiden, welche Regel sich durchsetzt; die Entwicklung ist normalerweise pfadabhängig; es gibt Lock-ins; die über die Effizienz richtende Umwelt wird selbst wiederum in der Evolution verändert; u.a.m. ${ }^{28}$

Da der Markttest somit ungeeignet ist, über die Effizienz oder Ineffizienz der Mitbestimmung zu entscheiden, sind empirische Untersuchungen erforderlich, um mehr Klarheit darüber zu gewinnen, in welchem Maße die diskutierten potentiellen Effizienzvorteile der Mitbestimmung genutzt wurden und inwieweit die befürchteten negativen Konsequenzen eingetreten sind. Im folgenden Kapitel wollen wir uns daher einigen empirischen Befunden zuwenden. weitere, allerdings wenig stichhaltige, Argumente gegen die spontane Entwicklung einer effizienten mitbestimmten Unternehmensverfassung finden sich bei Smith, S.C., JEBO. 


\section{Erfahrungen mit der Mitbestimmung in theoretischer Perspektive}

\section{Befunde aus ökonometrisch orientierten Studien}

Kornelius Kraft (1989) hat in seiner informativen Übersicht die empirischen Studien zur Mitbestimmung in zwei große Gruppen eingeteilt: Die erste Kategorie von Untersuchungen kennzeichnet er als "verhaltensorientiert" (behavioral approach), und zwar aus dem Grunde, weil sie - vor allem mit Mitteln der empirischen Sozialforschung das Verhalten von Unternehmensleitung, Betriebsrat, Beschäftigten usw. unter den Bedingungen gesetzlicher Mitbestimmung untersuchen. Die zweite - ökonometrisch orientierte - Gruppe von Untersuchungen befaßt sich mit den quantitativen Wirkungen der Mitbestimmung auf Produktivität, Löhne, Rentabilität und andere ökonomische Größen. Während die "verhaltensorientierten" Studien zu den Wirkungen betrieblicher und unternehmensbezogener Mitbestimmung nunmehr seit rund $40 \mathrm{~J}$ ahren durchgeführt werden, gibt es ernstzunehmende ökonometrische Untersuchungen erst seit Beginn der 80er Jahre. Die ersten Studien von Svejnar, J. (RES; EER; Codetermination) befassen sich allerdings noch nicht mit den Wirkungen des Mitbestimmungsgesetzes von 1976 (zu jenem Zeitpunkt wäre wohl auch die empirische Basis für ernsthafte ökonometrische Untersuchungen in diesem Bereich viel zu schmal gewesen), sondern sie untersuchen vor allem die Wirkungen der Montan-Mitbestimmung auf Lohnhöhe und Produktivität. Die Ergebnisse seiner Untersuchungen lassen sich vereinfacht dahingehend zusammenfassen, daß ein signifikanter Einfluß von Montan-Mitbestimmung (und Betriebsverfassungsgesetz 1972) nicht festzustellen ist. ${ }^{29}$

Die erste ökonometrische Studie, die nicht nur den Wirkungen des Montan-Mitbestimmungsgesetzes 1951, sondern auch des Mitbestimmungsgesetzes von 1976 gewidmet war, stammt von Benelli,G./Loderer, C./Lys, T. (1987). Ihre Hypothesen bezüglich der Wirkungen zur Mitbestimmung, wie verringerte Dividendenzahlungen, höhere Kapitalintensität und eine Veränderung des Investitionsverhaltens, finden jedoch in ihren sektoralen und unternehmensbezogenen Daten letztlich keine Bestätigung. ${ }^{30}$ Auch eine neuere Studie von Gurdon, M./Rai, A. (1990) kommt letztlich zu keinen schlüssigen Ergebnissen. Sie untersuchen (auf der Grundlage von Fragebogenergebnissen aus 63 westdeutschen Unternehmen) alternativ einerseits die Hypothese der Eigentumsrechtstheoretiker, daß unter Bedingungen der Mitbestimmung kapitalintensiver produziert wird, und andererseits die Erwartung der "Partizipationstheoretiker", daß die Produktivität mitbestimmter Unternehmen steigen wird. Die Ergebnisse dieser Studie zeigen zwar, daß bei den untersuchten mitbestimmten Unternehmen eine Senkung des Kapital-Arbeit-Einsatzverhältnisses festzustellen war und daß die Rentabilität zunahm, während gleichzeitig eine Abnahme der Produktivität registriert wurde. Aus verschiedenen Gründen - nicht zuletzt wegen der schmalen empirischen Basis 282-287. Hingegen gilt als ziemlich sicher - wie Gerlach, K./Schmidt, E.M. empirisch gezeigt haben -, daß die Lohnhöhe positiv mit der Unternehmensgröße - und auf Großunternehmungen ausschließlich bezieht sich ja die Mitbestimmung - korreliert. 
muß jedoch dieses Ergebnis nur als sehr vorläufig betrachtet werden. Gurdon und Rai folgern selbst: "The weight of the evidence slightly favors the participation theorists, although it is far from conclusive" (S. 289).

Für die Beurteilung der ökonomischen Wirkungen der Mitbestimmung wären in der Tat weitere ökonometrische Untersuchungen, wie sie z. B. für verschiedene Formen der Gewinnbeteiligung bereits vorliegen (vgl. z. B. Blinder 1990), von größter Bedeutung. ${ }^{31}$

\section{Flucht aus der Mitbestimmung?}

Da einerseits die ökonometrischen Studien, wie dargelegt, bisher zu keinen schlüssigen Ergebnissen geführt haben und andererseits die verhaltenswissenschaftlich orientierten Untersuchungen ernsthaften methodischen Bedenken ausgesetzt sind (vgl. dazu unten III.), bietet sich zunächst eine relativ unaufwendige, wenn auch nur als Vorklärung nützliche Überprüfung der Wirkungen der "voice option" Mitbestimmung seitens der Arbeitnehmer dadurch an, daß man die Wahrnehmung oder auch Nicht-Wahrnehmung der "exit option" Flucht aus der Mitbestimmung seitens der Anteilseigner untersucht. Nach den gesetzlichen Bestimmungen unterliegen ja nur Kapitalgesellschaften mit mehr als 2.000 Beschäftigten in der Bundesrepublik Deutschland einer derartigen gesetzlichen "Einschränkung". Die Eigentümer haben also die Möglichkeit, sich durch allerdings nicht kostenlose - Abwanderung aus dem Geltungsbereich des Gesetzes seinen mutmaßlich negativen Wirkungen zu entziehen. Dies kann geschehen durch Rechtsformwechsel (Übergang zu einer Personengesellschaft), durch Aufspaltung des Unternehmens in kleinere Einheiten, die weniger als 2.000 Arbeitnehmer beschäftigen, oder auch durch Verlagerung ins Ausland. Tatsächlich war in der Diskussion um die Wirkungen des Mitbestimmungsgesetzes in den 70er Jahren die vermutete "Flucht aus der Mitbestimmung" ein wesentliches Thema. Die Verabschiedung des Mitbestimmungsgesetzes 1976 und die Bestätigung seiner Verfassungsmäßigkeit 1979 haben in der Tat auch in einzelnen Fällen zu derartigen Ausweichreaktionen geführt. Obwohl es sich nur um Einzelfälle handelte, sahen auch die Gewerkschaften hierin eine nicht unbedeutende Gefahr, und es wurden juristische Vorschläge in die öffentliche Diskussion gebracht, die eine derartige Flucht aus der Mitbestimmung durch Rechtsformzwang oder auch durch Ausdehnung des Geltungsbereichs dieser gesetzlichen Regelung auf andere Unternehmensformen vorsahen (vgl. Nagel, B.: Unternehmensmitbestimmung, S. 162-165).

Tatsächlich haben sich Erwartungen und Befürchtungen an die Wahrnehmung dieser "exit option" nicht erfüllt. Die Zahl der mitbestimmten Unternehmen ist von 472 aufgestellt, das sowohl zeitliche als auch sektorale Vergleiche mit einschließt und das er derzeit in Kooperation mit anderen Wissenschaftlern empirisch umzusetzen bemüht ist. Hierzu liegen aber gegenwärtig noch keine publikationsreifen Ergebnisse vor. Die Verfasser dieses Aufsatzes verfügen lediglich über mündliche Mitteilungen von Kornelius Kraft. 
Firmen (1978) auf 522 Unternehmen (1989; vgl. Kronenberg, B./Scheiber-Lange, I.) und im Folgejahr auf 544 Gesellschaften (1990; Informationsdienst des Instituts der Deutschen Wirtschaft 1991) gestiegen. Per Saldo hat sich also deren Zahl um 72 erhöht. Dabei zeigen Detailanalysen, daß seit 1978 insgesamt 110 Unternehmen aus dem Geltungsbereich dieses Gesetzes ausgeschieden sind, vor allem durch Zusammenschlüsse zu noch größeren Unternehmen; demgegenüber spielten Konkurse oder die Verkleinerung der Belegschaften unter die Mitbestimmungsschwelle von $2.000 \mathrm{Be}$ schäftigten nur eine geringfügige Rolle. Gleichzeitig wurden 182 Unternehmen neu mitbestimmungspflichtig (vgl. Informationsdienst des Instituts der deutschen Wirtschaft). Auch in der öffentlichen Diskussion von Arbeitgeber- und Gewerkschaftsseite spielt die "Flucht aus der Mitbestimmung" schon seit längerem keine Rolle mehr. Dies deutet ganz offensichtlich darauf hin, daß die Wahrnehmung dieser Option durch die Kapitaleigner zumindest derzeit nicht ernsthaft in Betracht gezogen wird, so daß auch entsprechende Gegenreaktionen etwa durch Gesetzgebungsvorschläge seitens der Gewerkschaften ausbleiben.

Neben den oben erwähnten Möglichkeiten, sich dem Geltungsbereich des Mitbestimmungsgesetzes von $1976 \mathrm{zu}$ entziehen, gibt es auch noch die Option der "kleinen Fluchten", vor allem durch eine rigide Beschränkung der zustimmungspflichtigen Geschäfte, die dem (mitbestimmten) Aufsichtsrat zur Genehmigung vorzulegen sind. In der Tat zeigen empirische Untersuchungen, daß derartige Bestrebungen und Praktiken vor allem in eigentümerkontrollierten Kapitalgesellschaften (Steinmann, H. et al.: Trennung) sowie in Unternehmen mit hohem ausländischem Kapitalanteil (Steinmann, H. et al., ZfB) festzustellen sind. In der Regel handelt es sich aber bei einer restriktiven Handhabung der Aufsichtsratskontrolle um eine bereits vor 1976 bestehende - und nicht etwa aufgrund des Gesetzes eingeführte Praxis; im Gegenteil, es gelang den Arbeitnehmervertretern im Aufsichtsrat in einigen Fällen sogar, den Umfang der zustimmungspflichtigen Geschäfte zu vergrößern (vgl. Bamberg, U. et al., Abschnitt B).

Allerdings läßt dieser wohl ziemlich eindeutige empirische Befund zumindest noch zwei wichtige theoretische Fragen offen:

(1) Ist die Wahrnehmung dieser Option in nennenswertem Umfang deswegen unterblieben, weil die Transaktionskosten einer "Flucht aus der Mitbestimmung" verglichen mit den "Kosten der Mitbestimmung" - einfach prohibitiv hoch waren?

(2) Sind Ausweichreaktionen - wie Rechtsformwechsel, Verkleinerung oder Abwanderung ins Ausland - einfach deswegen unterblieben, weil die Regelungen des Mitbestimmungsgesetzes nicht dazu geeignet sind, der Arbeitnehmerseite und den Gewerkschaften einen auch nur irgendwie bedeutenden Einfluß auf die Entscheidungen des Unternehmens einzuräumen? Etwas überspitzt gefragt: Hat die Unternehmensmitbestimmung deswegen keine Wirkungen gezeigt, weil sie ohnehin wirkungslos ist?

Diese Frage läßt sich zum gegenwärtigen Zeitpunkt nicht eindeutig beantworten; einige vorläufige Überlegungen hierzu wollen wir jedoch abschließend anhand einiger ausgewählter Befunde aus den verhaltensorientierten Untersuchungen der letzten zehn 
Jahre anstellen.

\section{Befunde aus verhaltensorientierten Studien}

Auch die Ergebnisse der verhaltensorientierten Studien zum Mitbestimmungsgesetz 1976 sind keineswegs schlüssig. Auf der Grundlage einer Befragung von Bankfachleuten und Gewerkschaften einerseits, von Unternehmensleitungen und Betriebsräten andererseits kam das von Witte, E. (Einflußpotential; Einfluß; ZfbF 1981; ZfbF 1982) geleitete Forschungsteam zur Konstruktion eines Maßes für den Einfluß der Beschäftigten in den untersuchten Unternehmungen (vgl. im einzelnen Welchowski, P.). Ein bemerkenswertes Ergebnis dieser Untersuchungen ist der Befund, daß die Unternehmensleitung im allgemeinen den Beschäftigten weniger Einfluß beimißt, als dies die Betriebsratsmitglieder tun. Eine signifikante Korrelation zwischen dem quantitativ gemessenen Einflußpotential der Beschäftigten und der Höhe von Gewinn und cash flow der untersuchten Unternehmungen konnte nicht festgestellt werden. Die konkurrierenden Hypothesen, daß Unternehmensmitbestimmung zu einer Erhöhung oder aber zu einer Senkung der Rentabilität in den betroffenen Unternehmen führt, wurden also durch diese Untersuchungen gleichermaßen nicht bestätigt. Auch ein signifikanter Zusammenhang zwischen dem Einflußpotential der Beschäftigten und dem unternehmerischen Handlungsspielraum konnte nicht festgestellt werden (Witte, E.: Einfluß). Ein zeitlicher Vergleich der befragten Unternehmen vor und nach Einführung des Mitbestimmungsgesetzes 1976 ergab ebenfalls keine eindeutige Antwort auf die Frage, ob das Einflußpotential der Beschäftigten nach Einführung des Gesetzes zuoder abgenommen hat.

$\mathrm{Zu}$ ähnlichen Ergebnissen kommen auch Kirsch, W./Scholl, W., Scholl, W. und Paul, G. Aufgrund einer detaillierten Befragung von Unternehmen zu ihrer Personal- und Investitionspolitik konstruierten sie einen Index des Handlungsspielraums für das Management in Unternehmen, die teils der paritätischen Montan-Mitbestimmung, teils der Drittelparität nach dem Betriebsverfassungsgesetz 1952 und teilweise der Quasi-Parität des Mitbestimmungsgesetzes von 1976 unterlagen. Sie gelangen u.a. zu dem überraschenden Resultat, daß der unternehmerische Handlungsspielraum mit zunehmenden Mitbestimmungsrechten ebenfalls zunimmt. Ein wesentliches Argument zur Erklärung dieses zunächst überraschenden Ergebnisses ist nach Scholl, W./Blumschein, $\mathrm{H}$. die Tatsache, daß sich Mitbestimmung positiv auf die Bedürfnisbefriedigung der Arbeitnehmer auswirkt, und zwar vor allen Dingen deswegen, weil

"die Arbeitnehmer ... bei ungünstiger wirtschaftlicher Lage von der Mitbestimmung profitieren, wenn der Betriebsrat großen Einfluß hat. Bei guter wirtschaftlicher Situation nützt das Management seine Handlungsmöglichkeiten im Sinne einer Marketing-Strategie und sorgt für eine hohe Bedürfnisbefriedigung der Arbeitnehmer, wobei das Zurückdrängen des Einflusses des Betriebsrates vermutlich ein Motiv dafür ist, unabhängig davon, ob sich dies auch verwirklichen läßt ... Die Mitbestimmungssituation eröffnet ein konkurrierendes Marketing zwischen Management und Arbeitnehmervertretern, die beide durch 
Leistungen für die Arbeitnehmer Unterstützung erhalten wollen. Dabei ist ein erfolgreiches Marketing des Betriebsrates gegenüber den Arbeitnehmern gebunden an Erfolge bei der Partizipation von Arbeitnehmervertretern an unternehmenspolitischen Entscheidungen. Da Mitbestimmung erst ein solches konkurrierendes, innerbetriebliches Marketing hervorruft, ist Mitbestimmung für die Bedürfnisbefriedigung der Arbeitnehmer nicht nur bei erfolgreicher Partizipation der Arbeitnehmervertreter von Vorteil, sondern auch dann, wenn diese Bedürfnisbefriedigung primär dem Marketing des Managements zuzuschreiben ist, da es ohne die Konkurrenz zu den Arbeitnehmervertretern vermutlich nicht im selben Maße sich dazu genötigt sehen würde" (Scholl, W./Blumschein, H., S. 294 f.).

Die Befunde der Forschergruppe um Kirsch deuten darauf hin, daß Mitbestimmung keine reine Umverteilung darstellt, sondern möglicherweise ein "Spiel" mit einer positiven Auszahlung an alle beteiligten Parteien. Die bisher vorgelegten Befunde reichen natürlich keineswegs dazu aus, um Mitbestimmung in Unternehmen - analog zu Markttausch zwischen gleichberechtigten Partnern - als kooperatives Spiel zu betrachten, bei dem - trotz einer gewissen Umverteilung der Ausgangsausstattung an Eigentumsrechten - letztlich alle beteiligten Parteien profitieren. Immerhin erscheint dieses Szenario als mindestens ebenso plausibel wie die Sicht der Eigentumsrechtstheoretiker, die neben einer Umverteilung auch noch eine Wohlfahrtsverschlechterung vermuten.

Wie die Darstellung der juristischen Grundlagen der Unternehmensmitbestimmung in Abschnitt A.II. oben deutlich gemacht hat, sind durch das Mitbestimmungsgesetz von 1976 keine wesentlichen Entscheidungsrechte an die Beschäftigten delegiert worden. In einer undifferenzierten Betrachtung könnte man sogar zu der Auffassung neigen, daß die Quasi-Parität des Mitbestimmungsgesetzes von 1976 keine bedeutende Veränderung gegenüber der Drittelbeteiligung nach dem Betriebsverfassungsgesetz 1952 mit sich bringt. Dieser Schluß erscheint jedoch vor allem im Hinblick auf die Information der Arbeitnehmerseite voreilig.

Auch wenn die vorliegenden ökonometrischen und verhaltensorientierten Studien keine eindeutigen Schlüsse zulassen, so deuten doch einige Befunde darauf hin, daß sich die Position der Arbeitnehmerseite zumindest bei der Gewinnung und Weitergabe von Informationen verbessert hat; dies gilt insbesondere deswegen, weil es in der Praxis eine enge Kooperation, häufig sogar in Form einer personellen Identität zwischen dem Betriebsrat, den Mitgliedern des Wirtschaftsausschusses und den Arbeitnehmervertretern im Aufsichtsrat mitbestimmter Unternehmen gibt. Diese Zusammenarbeit führt wohl nicht zu dem von Prosi prognostizierten "Kumulationseffekt", aber doch immerhin zur Möglichkeit einer effektiveren Wahrnehmung von Informationsrechten. Zwar bestanden diese Rechte im Prinzip bereits vor 1976; ihre effektive Wahrnehmung ist jedoch auch unter den Bedingungen einer Quasi-Parität günstiger als bei einer reinen Drittelbeteiligung, nicht zuletzt deswegen, weil die Anteilseignerseite nicht ohne langfristig negative Folgen für die Kooperation mit Betriebsrat und Gewerkschaften 
permanent das Doppelstimmrecht des Aufsichtsratsvorsitzenden einsetzen kann, um einseitig ihre Vorstellungen durchzusetzen. Selbst das am Landesinstitut Sozialforschungsstelle Dortmund durchgeführte Forschungsprojekt (Bamberg, U. et al.), das aus gewerkschaftsnaher Perspektive dem Mitbestimmungsgesetz sehr kritisch gegenübersteht, sieht hier durchaus einige Verbesserungen und Handlungsmöglichkeiten. Auch die vorliegenden empirischen Befunde zur Praxis des Mitbestimmungsgesetzes sprechen dafür, daß im allgemeinen eher auf Konsensbildung im (mitbestimmten) Aufsichtsrat hingewirkt wird als auf eine Durchsetzung der faktischen Überparität der Anteilseignerseite. Die im Aktiengesetz postulierte Auskunftspflicht des Vorstands an den Aufsichtsrat ist dabei auch als ein Mittel zu sehen, die Zustimmung der Arbeitnehmerseite - insbesondere zu unpopulären Maßnahmen - zu erreichen. Selbstverständlich ist den beteiligten Parteien, wenn sie konsensfähige "Pakete" schnüren, durchaus bewußt, daß im Konfliktfall letztlich die Position der Anteilseignerseite gegen die Arbeitnehmerbank durchgesetzt werden kann; aber in solchen "Paketen" kommen wohl doch Arbeitnehmerinteressen besser zur Geltung als im Falle der Drittelbeteiligung nach dem Betriebverfassungsgesetz 1952, in dem der faktische Zwang zur Einigung viel geringer ist als unter den Bedingungen der Quasi-Parität.

Wenn die Arbeitnehmervertreter im Aufsichtsrat ihre Auskunftsrechte gegenüber dem Vorstand entschlossen wahrnehmen - und dieser nicht nur wegen der juristischen Verpflichtungen, sondern auch im Interesse einer Konsensbildung im Aufsichtsrat diesen Ansprüchen weitgehend entgegenkommt -, so können dadurch auch positive Externalitäten für die Anteilseigner wie auch für die Fremdkapitalgeber (Banken usw.) entstehen: Die Wahrnehmung von Mitbestimmungsrechten kann also zugleich die Kontrollfunktion des Aufsichtsrats insgesamt gegenüber dem Vorstand verbessern und damit zugleich den Interessen der Kapitalgeber dienen. Daß nach den Befunden von Steinmann, H. et al. (Trennung) der Umfang zustimmungspflichtiger Geschäfte in managerkontrollierten Unternehmen in der Regel sehr erheblich ist, spricht auch dafür, daß derartige Kontrollmöglichkeiten des (mitbestimmten) Aufsichtsrates tatsächlich auch wahrgenommen werden.

Eine weitere Verbesserung der Informationsflüsse kann auch durch die von Bamberg, U. et al. festgestellte "distanzierte Kooperation" der - oftmals der Anteilseignerseite zuzurechnenden - Arbeitsdirektoren mit der Arbeitnehmerseite und den Gewerkschaften darstellen. Es ist zumindest plausibel anzunehmen - aber weiterer empirischer Forschung bedürftig -, daß die Arbeitsdirektoren eher an einer Konsensbildung mit dem Betriebsrat (insbesondere dessen Vorsitzenden) als an ständiger Konfrontation mit der Arbeitnehmerseite und den Gewerkschaften interessiert sind. Darauf deuten z. B. auch die von uns durchgeführten Fallstudien in der Automobilindustrie (Diefenbacher, H. et al.) und in der elektrotechnischen Industrie (Nutzinger, H.G. et al.) hin.

\section{E. Schluß}

Obwohl sowohl die ökonometrischen als auch die verhaltensorientierten Studien bisher keine schlüssigen Ergebnisse über die Wirkungen der Unternehmensmitbestimmung nach dem Gesetz von 1976 erbracht haben, sprechen die vorliegenden Befunde doch 
eher gegen die Annahme einer verringerten Anpassungsfähigkeit mitbestimmter Unternehmen als für die Akzeptierung dieser vor allem von den amerikanischen Eigentumsrechtstheoretikern vorgebrachten Hypothese. Die von den "Partizipationstheoretikern" mit der Mitbestimmung verbundenen positiven Erwartungen sind allerdings bisher gleichfalls nicht zwingend belegt. Ob dies daran liegt, daß die Einflußmöglichkeiten nach dem Mitbestimmungsgesetz 1976 insgesamt zu gering sind, um zu einem merklich veränderten Unternehmensverhalten mit entsprechend empirisch feststellbaren Konsequenzen zu führen, oder ob eine zweifelsfreie Ermittlung durchaus vorhandener Wirkungen bisher daran scheiterte, daß es noch keine zureichenden empirischen Untersuchungen gibt, muß beim gegenwärtigen Forschungsstand noch als offene Frage betrachtet werden.

Allerdings deuten die oben vorgebrachten theoretischen Argumente darauf hin, daß in einer Welt, die durch Informationsunvollkommenheiten, Motivationsschwäche und Ressourcenspezifitäten gekennzeichnet ist, Mitbestimmung ein Mittel sein kann, die daraus hervorgerufenen Effizienz- und Verteilungsprobleme zu mildern. Argumente, die die Schädlichkeit der Mitbestimmung nachweisen, beziehen sich demgegenüber meist auf eine vollkommene Welt, in der die Mitbestimmungsregelungen als Beschränkungen erscheinen, die die Erreichung optimaler Zustände verhindern. Berücksichtigt man neben den empirischen Befunden diese theoretischen Erkenntnisse, so spricht sehr viel für Mitbestimmung. 


\section{Literatur}

Alchian, A. A.

Alchian, A.A.

Demsetz, $\mathrm{H}$.

Aoki, M.

Aoki, M.

Arrow, K.J.

Backhaus, J.

Bamberg, U., Bürger, M., Mahnkopf, B., Martens, $\mathrm{H}$., Tiemann, J.

Benelli, G./Loderer, C./Lys, T.

Biehler, H., Brandes, W./Buttler, F./Gerlach, K./Liepmann, P.

Blien, U.

Blinder, A.S. (Hrsg.)

Boyd, R./Richerson, $P$.
Specificity, Specialization, and Coalitions. Zeitschrift für die gesamte Staatswissenschaft 140 (1984), 34 ff. (Alchian, A.A., $\mathrm{ZgS})$

Production, Information Costs, and Economic Organization American Economic Review 62 (1972) 777 ff. (Alchian, A.A./Demsetz, H., AER)

The Cooperative Game Theory of the Firm, 1984 (Aoki, M.: Firm)

Information, Incentives, and Bargaining in the Japanese Economy, 1988 (Aoki, M.: Information).

The Limits of Organization, 1974 (Arrow, K.J.)

Mitbestimmung im Unternehmen. Eine ökonomische Rechtsanalyse des Verfassungsgerichtsurteils vom 1. März 1979 als Beitrag zur Theorie der wirtschaftlichen Rechtspolitik, 1987 (Backhaus, J.)

Aber ob die Karten voll ausgereizt sind ...: Zehn Jahre Mitbestimmungsgesetz 1976 in der Bilanz, 1987 (Bamberg, U. et al.)

Labor Participation in Corporate Policy-making Decisions : West Germany's Experience with Codetermination, Journal of Business 60, (1987), $553 \mathrm{ff}$. (Benelli, G. et al., JoB)

Arbeitsmarktstrukturen und -prozesse, 1981 (Biehler, H. et al.)

Unternehmensverhaltenund Arbeitsmarktstruktur, 1986(Blien, U.)

Paying for Productivity - A Look at the Evidence, 1990 (Blinder, A.S.).

Culture and the Evolutionary Process, 1985 (Boyd, R./ Richerson, P.) 
Brandes, W./Weise, $P$.

Brandes, W./Buttler F./Dorndorf, E.

Bundesminister

Coase, R.H.

Diefenbacher, H., Kißler, L./Nutzinger, H.G./Teichert, V.

Dorndorf, E.

Downs, A.

Edwards, R.

Eger, T./Weise, P.

P.)

Arbeitsmarkttheorie und Arbeitsrechtswissenschaft: Analoge Probleme und Diskussionsschwerpunkte im Hinblick auf die Funktionsfähigkeit der Arbeitsmärkte, in: Währungsreform und soziale Marktwirtschaft. Erfahrungen und Perspektiven nach 40 Jahren (Schriften des Vereins für Socialpolitik, Gesellschaft für Wirtschafts- und Sozialwissenschaften, Neue Folge Band 190), 1989, 489 ff. (Brandes, W. et al.)

für Arbeit und Sozialordnung, Mitbestimmung. Mitbestimmungs-Gesetz, Montan-Mitbestimmung, Betriebsverfassung, Mitbestimmungs-Urteil des Bundesverfassungsgerichts, 1979 (Bundesminister für Arbeit und Sozialordnung)

The Nature of the Firm. Economica NS. 16 (1937), 386 ff. (Coase, R.H., Economica)

Mitbestimmung: Norm und Wirklichkeit. Fallstudie aus einem Großbetrieb der Automobilindustrie, 1984 (Diefenbacher, H. et al.)

Vertragsdurchsetzung als Funktion des Kündigungsschutzes. Zeitschrift für Arbeitsrecht 20 (1989), $345 \mathrm{ff}$. (Dorndorf, E., ZfA)

Non-Market Decision Making. A Theory of Bureaucracy. American Economic Review 55 (1965), 439 ff. (Downs, A., AER)

Contested Terrain, 1979 (Edwards, R.)

Grundlagen einer ökonomischen Theorie der Partizipation, in: Diefenbacher, H./Nutzinger, H.G. (Hrsg.), Mitbestimmung: Theorie, Geschichte, Praxis, 1984, 39 ff. (Eger, T./Weise, P.: Grundlagen)

Eger, T./Weise, P. Die Evolution kapitalistischer und laboristischer Unternehmungen als Prozeß der Selbstorganisation, in: FitzRoy, F.R./Kraft, K. (Hrsg.), Mitarbeiterbeteiligung und Mitbestimmung im Unternehmen, 1987, 295 ff. (Eger, T./Weise, P.: Evolution) 
Eger, T./Weise, P. Participation and Codetermination in a Perfect and an Imperfect World, in: Nutzinger, H.G./Backhaus, J. (1989), $11 \mathrm{ff}$. (Eger, T./Weise, P.: Participation)

Fama, E.F.

Agency Problems and the Theory of the Firm. Journal of Political Economy 88 (1980), 288 ff. (Fama, E.F., JPE)

FitzRoy, F./Mueller Cooperation and Conflict in Contractual Organizations.

D.C.

Quarterly Review of Economics and Business 24 (1984), $24 \mathrm{ff}$.

(FitzRoy, F./Mueller, D.C., QREB)

Fox, A.

Beyond Contract: Work, Power, and Trust Relations, 1974 (Fox, A.)

Furubotn, E.

Codetermination and the Modern Theory of the Firm: A Property Rights Analysis. Journal of Business 61 (1988), 165 ff. (Furubotn, E., JoB)

Furubotn, E. $\quad$ A General Model of Codetermination, in: Nutzinger, H.G./Backhaus, J. (1989), 41 ff. (Furubotn, E.: General Model)

Gerlach, K./Schmidt Unternehmensgröße und Entlohnung. Mitteilungen aus der ArE.M. beitsmarkt- und Berufsforschung 22 (1989), 355 ff. (Gerlach, K./Schmidt, E.M.)

Georgescu-Roegen, N. Energy and Economic Myths. Institutional and Analytical Economic Essays, 1976 (Georgescu-Roegen, N.)

Graumann, C.F. Sozialpsychologie, 2. Halbband, 1972 (Graumann, C.F.) (Hrsg.)

Gurdon, M./Rai, A. Codetermination and Enterprise Performance: Empirical Evidence from West Germany. Journal of Economics and Business 42 (1990) 289 ff. (Gurdon, M./Rai, A., JEB)

Hirschman, A.O. Abwanderung und Widerspruch, (Schriften zur Kooperationsforschung, A. Studien, Band 8), 1974 (Hirschman, A.O.)

Informationsdienst des Instituts der deutschen Wirtschaft, Mitbestimmungsgesetz 1976. Starke Position der IG Metall, iwd 42 (1991) 2 (Informationsdienst des Instituts der deutschen Wirtschaft)

Jensen, M./MeckRights and Production Functions: An Application to Labor ling, $\mathrm{W}$. managed Firms and Codetermination. Journal of Business 52 (1979), 469 ff. (Jensen, M./Meckling, W., JoB) 
Kappler, E.

Kirsch, W./Scholl, W.

Knight, F.H.

Kraft, $\mathrm{K}$.

Kraft, K.

Kronenberg, B., Scheiber-Lange, I.

Leibenstein, $\mathrm{H}$.

Leibenstein, $\mathbf{H}$.

Leibenstein, $\mathrm{H}$.

Marglin, St.A.

MacLeod, W.B.

McCain, R.A.

McCain, R.A.
Ökonomische Beurteilung der Mitbestimmung, Manuskript, Universität-Gesamthochschule Wuppertal, 1978 (Kappler, E.)

Was bringt die Mitbestimmung: Eine Gefährdung der Handlungsfähigkeit und/oder Nutzen für die Arbeitnehmer? Die Betriebswirtschaft 43 (1983), 541 ff. (Kirsch, W./Scholl, W.)

Risk, Uncertainty, and Profit, 1921 (Knight, F.H.)

Partizipation und interner Arbeitsmarkt. Wechselwirkung zwischen Humankapital und Mitarbeiterbeteiligung, 1984 (Kraft, K.: Partizipation)

Empirical Studies on Codetermination: A Selective Survey and Research Design, in: Nutzinger, H.G./Backhaus, J. (1989), $277 \mathrm{ff}$. (Kraft, K.: Empirical Studies)

Mitbestimmungsunternehmen nach dem Stand vom 31. Dezember 1989, WSI-Mitteilungen 7/1990, 468-476 (Kronenberg, B./Scheiber-Lange, I.)

The Prisoners' Dilemma in the Invisible Hand: An Analysis of Intrafirm Productivity. American Economic Review, P and P, 72 (1982), 92 ff. (Leibenstein, H., AER 1982)

Property Rights and X-Efficiency: Comment, American Economic Review 73 (1983), 831 ff. (Leibenstein, H., AER 1983)

Inside the Firm - The Inefficiencies of Hierarchy, 1987 (Leibenstein, H.: Firm).

What Do Bosses Do? The Origins and Functions of Hierarchy in Capitalist Production. Review of Radical Political Economics 6 (1974), 60 ff. (Marglin, St.A., RRPE)

Equity, Efficiency, and Incentives in Cooperative Teams, in: Advances in the Economic Analysis of Participatory and Labor Managed Firms, Vol. 3 (1988), 5 ff. (MacLeod, W.B.)

A Theory of Codetermination. Zeitschrift für Nationalökonomie 40 (1980), 65 ff. (McCain, ZfN)

Codetermination, Collective Bargaining, Commitment, and Sequential Games, in: Nutzinger, H.G./Backhaus, J. (1989), 103 ff. (McCain, R.A.: Codetermination) 
Nagel, B. Unternehmensmitbestimmung. Eine problemorientierte Einführung, 1980 (Nagel B.: Unternehmensmitbestimmung)

Nagel, B. Paritätische Mitbestimmung und Grundgesetz, 1988 (Nagel, B.: Paritätische Mitbestimmung)

Nutzinger, H.G. The Firm as a Social Institution: The Failure of the Contractarian Viewpoint, in: Backhaus, J./Eger, T./Nutzinger, H.G. (Hrsg.), Partizipation in Betrieb und Gesellschaft, 1978, $45 \mathrm{ff}$. (Nutzinger, H.G.: Firm)

Nutzinger, H.G. Codetermination in West Germany: Institutions and Experiences, in: Nutzinger, H.G./Backhaus, J. (1989), 163 ff. (Nutzinger, H.G.: Codetermination)

Nutzinger, H.G., Codetermination. A Discussion of Different Approaches, Backhaus, J. (Hrsg.) 1989 (Nutzinger, H.G./Backhaus, J.)

Nutzinger, H.G., Mitbestimmung in der Krise. Fallstudie aus einem GroßSchasse, U./

Teichert, V. betrieb der elektrotechnischen Industrie.

1987 (Nutzinger, H.G. et al.)

Pagano, U.

Property Rights, Asset Specifity, and the Division of Labour under Alternative Capitalist Relations. Cambridge Journal of Economics, 15 (1991), 315 ff. (Pagano, U., CJE)

Pagano, U. Property Rights, Equilibria, and Institutional Stability, in: Backhaus, J. (Hrsg.), Systemwandel und Reform in östlichen Wirtschaften, 1991, 185 ff. (Pagano, U.: Institutional Stability)

Paul, G. Mitbestimmung im Aufsichtsrat - Eine vergleichende empirische Studie, in: Nutzinger, H.G. (Hrsg.), Mitbestimmung und Arbeiterselbstverwaltung. Praxis und Programmatik, 1982, 299 ff. (Paul, G.)

Pejovich, S. $\quad$ Codetermination: A New Perspective for the West, in: ders. (Hrsg.), The Codetermination Movement in the West, 1978, 3 ff. (Pejovich, S.)

Prosi, G. Volkswirtschaftliche Auswirkungen des Mitbestimmungsgesetzes 1976, 1978 (Prosi, G.)

Putterman, L. On some recent explanation of why capital hires labor. Econo mic Inquiry 12 (1984), 171 ff. (Putterman, L., EI) 
Samuelson, P.A. Foundations of Economic Analysis, 1947 (Samuelson, P.A.)

Schmidtchen, D. $\quad$ "Sunk Costs", Quasirenten und Mitbestimmung, in: E. Böttcher u. a. (Hrsg.). Jahrbuch für Neue Politische Ökonomie, Bd. 6 (1987) 139 ff. (Schmidtchen, D., JNPÖ)

Scholl, W. Handlungsfähigkeit und Mitbestimmung, in: Diefenbacher, H./Nutzinger, H.G. (Hrsg.) Mitbestimmung: Theorie, Geschichte, Praxis, 1984, 229 ff. (Scholl, W.)

Scholl, W./Blum- Mitbestimmung und Bedürfnisbefriedigung der Arbeitnehmer, schein, $\mathrm{H}$. in: Nutzinger, H.G. (Hrsg.), Mitbestimmung und Arbeiterselbstverwaltung. Praxis und Programmatik, 1982, $267 \mathrm{ff}$. (Scholl, W./Blumschein, H.)

Smith, S.C. On the Economic Rationale for Codetermination Law. Journal of Economic Behavior and Organization 16 (1991), $261 \mathrm{ff}$. (Smith, S.C., JEBO)

Steinmann, H./Fees, Multinationale Unternehmen und Mitbestimmung. Zeitschrift W./Gerum, E. für Betriebswirtschaft 54 (1984), 368 ff. (Steinmann, H. et al., $\mathrm{ZfB})$

Steinmann, H./Fees, Trennung von Eigentum und Verfügungsgewalt und MitbeW./Gerum, E. stimmung, in: FitzRoy, F./Kraft, K. (Hrsg.), Mitarbeiterbeteiligung und Mitbestimmung im Unternehmen, 1987, $159 \mathrm{ff}$. (Steinmann, H. et al.: Trennung)

Stützel, W.

Preis, Wert und Macht. Analytische Theorie des Verhältnisses der Wirtschaft zum Staat. Nachdruck der Dissertation von 1952, 1972 (Stützel, W.)

Svejnar, J. Relative Wage Effects of Unions, Dictatorship and Codetermination: Econometric Evidence from Germany. Review of Economics and Statistics 63 (1981), 188 ff. (Svejnar, J., RES)

Svejnar, J. Employee Participation in Management, Bargaining Power and Wages. European Economic Review 18 (1982), $291 \mathrm{ff}$. (Svejnar, J., EER)

Svejnar, J. Codetermination and Productivity: Empirical Evidence from the Federal Republic of Germany, in: Jones, D.C./Svejnar, J. (Hrsg.), Participatory and Self-Managed Firms, 1982, $199 \mathrm{ff}$. (Svejnar, J.: Codetermination) 
Vogt, W.

Weise, P./Brandes, W.

Weise,P./Brandes W./Eger, T./Kraft, M.

Welchowski, P.

Williamson, O.E.

Witte, E.

Witte, E.

Witte, E.

Witte, E.

WSI-Projektgruppe
Theorie der kapitalistischen und einer laboristischen Ökonomie, 1986 (Vogt, W.)

A Synergetic View of Institutions. Theory and Decision 28 (1990), 173 ff (Weise, P./ Brandes, W.,TaD)

Neue Mikrökonomie, 2. Auflage, 1991 (Weise, P. et al.)

Mitbestimmung - Methodische Probleme der empirischen Einflußanalyse, in: Diefenbacher, H./Nutzinger, H.G. (Hrsg.), Mitbestimmung. Probleme und Perspektiven der empirischen Forschung, 1981 (Welchowski, P.)

The Economic Institutions of Capitalism, 1985 (Williamson O.E.)

Das Einflußpotential der Arbeitnehmer als Grundlage der Mitbestimmung. Die Betriebswirtschaft 40 (1980), $3 \mathrm{ff}$. (Witte, E.: Einflußpotential)

Der Einfluß der Arbeitnehmer auf die Unternehmenspolitik. Die Betriebswirtschaft 40 (1980), 541 ff. (Witte, E.: Einfluß)

Die Unabhängigkeit des Vorstandes im Einflußsystem der Unternehmung Zeitschrift für betriebswirtschaftliche Forschung 33 (1981), 273 ff. (Witte, E., ZfbF 1981)

Das Einflußsystem der Unternehmung in den Jahren 1976 und 1981. Zeitschrift für betriebswirtschaftliche Forschung 34 (1982), 416 ff. (Witte, E., ZfbF 1982)

Mitbestimmung in Unternehmen und Betrieb, 1981 (WSI-Projektgruppe) 